\title{
Comprehensive Analytical Study of the Greenhouse Effect of the Atmosphere
}

\author{
Peter Stallinga \\ University of the Algarve, FCT-DEEI, Faro, Portugal \\ Email: peter.stallinga@gmail.com
}

How to cite this paper: Stallinga, P. (2020) Comprehensive Analytical Study of the Greenhouse Effect of the Atmosphere. Atmospheric and Climate Sciences, 10, 40-80. https://doi.org/10.4236/acs.2020.101003

Received: November 20, 2019

Accepted: January 14, 2020

Published: January 17, 2020

Copyright $\odot 2020$ by author(s) and Scientific Research Publishing Inc. This work is licensed under the Creative Commons Attribution International License (CC BY 4.0).

http://creativecommons.org/licenses/by/4.0/

\begin{abstract}
Climate change is an important societal issue. Large effort in society is spent on addressing it. For adequate measures, it is important that the phenomenon of climate change is well understood, especially the effect of adding carbon dioxide to the atmosphere. In this work, a theoretical fully analytical study is presented of the so-called greenhouse effect of carbon dioxide. The effect of this gas in the atmosphere itself was already determined as being of little importance based on empirical analysis. In the current work, the effect is studied both phenomenologically and analytically. In a first attempt of energy transfer by radiation only, it is solved by ideal-gas-law equations and the atmosphere is divided into an infinite number of layers each absorbing and reemitting infrared radiation (surpassing the classical Beer-Lambert analysis of absorption). The result is that the exact structure of the atmosphere is irrelevant for the analysis; we might as well keep the two-box model for any analytical approach. However, the results are unsatisfactory in that they cannot explain the profile of the atmosphere. In a new approach, the atmosphere is solved by taking both radiative as well as thermodynamic processes into account. The model fully fits the empirical data and an analytical equation is given for the atmospheric behavior. Upper limits are found for the greenhouse effect ranging from zero to a couple of $\mathrm{mK}$ per $\mathrm{ppm} \mathrm{CO}_{2}$. It is shown that it cannot explain the observed correlation of carbon dioxide and surface temperature. This correlation, however, is readily explained by Henry's Law (outgassing of oceans), with other phenomena insignificant. Finally, while the greenhouse effect can thus, in a rudimentary way, explain the behavior of the atmosphere of Earth, it fails describing other atmospheres such as that of Mars. Moreover, looking at three cities in Spain, it is found that radiation balances only cannot explain the temperature of these cities. Finally, three data sets with different time scales (60 years, 600 thousand years, and 650 million years) show markedly different behavior, something that is inexplicable in the framework of the greenhouse theory.
\end{abstract}




\section{Keywords}

Greenhouse Effect, Thermodynamics, Radiation Balance, Correlation, Feedback, Water, Carbon Dioxide, Mars, Venus, Earth

\section{Introduction}

One of the strongest argument of Alarmists-those thinking that anthropogenic $\mathrm{CO}_{2}$ is significantly detrimental to the climate-is the correlation between the concentration of $\mathrm{CO}_{2}$ in the atmosphere (henceforth called $\left[\mathrm{CO}_{2}\right]$ ) and the temperature measured on Earth. Strong correlations between these two quantities were found in ice-core drilling records spanning some 600,000 years, made famous by the movie An Inconvenient Truth by Albertus Gore. Yet, as we know from statistics textbooks, "Correlation is not causation". In an earlier work [1], we analyzed this correlation and came to the conclusion that it is difficult to explain by the greenhouse effect, which can explain about $500 \mathrm{mK}$ for a doubling of $\mathrm{CO}_{2}$ in the atmosphere from $350 \mathrm{ppm}$ to $700 \mathrm{ppm}$; the climate sensitivity of carbon dioxide is $\mathrm{d} T / \mathrm{d}\left[\mathrm{CO}_{2}\right]=1.4 \mathrm{mK} / \mathrm{ppm}$, which we call the open-loop sensitivity $(s)$. It has to be noted that the correlation observed is about $95 \mathrm{mK} / \mathrm{ppm}$ [1], two orders of magnitude larger. To circumvent this problem, researchers introduced a positive feedback $\beta$ in the climate models, such that the overall sensitivity can be any value desired, $\mathrm{d} T / \mathrm{d}\left[\mathrm{CO}_{2}\right]=s /(1-s \beta)$, including the 95 $\mathrm{mK} / \mathrm{ppm}$ observed. The parameter $\beta$ has no justification other than that it can explain the data; it is retrodiction [2].

Some ad-hoc explanations are tried for feedback, such as the albedo effect, or outgassing of methane from ex permafrost regions. Note that we have rejected the idea of the ice-albedo effect being significant (in fact, Fresnel reflection hints at a reverse albedo effect; seawater is more reflective than ice at the grazing angles of sunlight at the poles [3]). It is however highly questionable that feedback is positive. If feedback were positive, the climate would have been out of control even without anthropogenic $\mathrm{CO}_{2}$; small perturbations would be self-amplified to infinity. That is, until the system saturates and by negative feedback settles at a stable value. The situation we are in, since it was not going anywhere rapidly, is that of a negative-feedback state. The value for $\beta$ found above is very close to the Barkhausen criterion for instability ( $\beta=1 / s$ ) which casts more doubt on the value and the positive feedback assumption [1]. Yet, positive feedback comes inevitably out of black-box supercomputer calculations, since they start with the assumption that temperature must result from carbon dioxide.

Negative feedback $\beta$, on the other hand, can equally easily be shown to result in an overall sensitivity that is smaller than the open-loop sensitivity, making it $s /(1+s \beta)$, which is smaller than $s$. We thus expect, on basis of physical laws, to have a climate sensitivity of $\mathrm{CO}_{2}$ smaller than $500 \mathrm{mK}$ for doubling of $\mathrm{CO}_{2}$ in the atmosphere. This may well be effectively unmeasurable. 
In this manuscript we take a look at the greenhouse effect with fully-analytical techniques, starting with physical laws, and avoid any computational tools, in an attempt to determine the climate variations caused by the greenhouse effect. This is the only viable method, since there is no planet B in which we can experiment. We will never know if theories (those that are coming out of black-box simulations) are correct, if they can never be tested or calibrated by reality. In such cases, instead of coming up with untestable theories, we have to revert to tested physical laws.

- Section 2: The greenhouse effect is phenomenologically introduced and compared to the alternative explanation for the data, namely Henry's Law. The observed correlations between temperature and $\mathrm{CO}_{2}$ are presented; these are the data we are going to attempt to explain.

- Section 3: A "classic" greenhouse model is presented where energy transfer is uniquely by radiation. First with the atmosphere as single body, then with the atmosphere as an infinite set of identical layers, and finally with a multi-layer model in which the atmosphere is in thermodynamic equilibrium, so that layers get thinner and colder upwards.

- Section 4: A thermodynamic-radiative model is presented in which each part of the atmosphere is in thermodynamic equilibrium and, moreover, exchanges heat not only by radiation, but also by other ways, such as convection, etc.

- Section 5 discusses some test cases of the model. They show mixed success.

- Section 6 augments the model by including feedback and secondary effects, such as water. It also tries to establish relaxations times of the system.

The parameters used in this work are given in Table 1.

\section{The Greenhouse Effect}

We want to begin by mentioning here that the "greenhouse effect" is a misnomer. That, because a real greenhouse does not work on the principle of that so-called greenhouse effect. We are led to believe that a greenhouse works on the principle of incoming radiation being in the visible range of radiation, for which glass (and the atmosphere) is transparent, while outgoing heat radiation is in wavelengths for which glass (and the atmosphere) is opaque. A real greenhouse, however, works on the principle that heated air is blocked from rising and thus stays trapped close to the surface. This can easily be shown by the fact that farmers, when they want to cool down their greenhouse, open windows at the ceiling, thus letting hot air out, while not changing the radiation configuration, see Figure 1. This immediately shows a fundamental difference between a real greenhouse and the atmosphere: a greenhouse is a closed system, with constant dimensions, whereas the atmosphere is an open-ended system, it is not limited by a ceiling that traps heat.

We also want to mention the curious fact that many farmers, in fact, inject $\mathrm{CO}_{2}$ into their greenhouses. Not to increase the temperature, but to increase crop yield. Photosynthesis is the reaction of carbon dioxide and water into sugar 
Table 1. Parameters, constants and variables used in this work.

\begin{tabular}{|c|c|c|c|}
\hline Symbol & Name & (Typical) Value & Unit \\
\hline$a$ & albedo (visible) & 0.306 & 1 \\
\hline$A$ & area & & $\mathrm{m}^{2}$ \\
\hline$\alpha$ & absorption coefficient & & $1 / \mathrm{m}$ \\
\hline$c$ & volumetric density & & (molecules) per $\mathrm{m}^{3}$ \\
\hline$C$ & areal density & & (molecules) per $\mathrm{m}^{2}$ \\
\hline$\left[\mathrm{CO}_{2}\right]$ & molar $\mathrm{CO}_{2}$ fraction & 350 & $10^{-6}(\mathrm{ppm})$ \\
\hline$c_{\mathrm{p}}$ & specific heat (constant $P$ ) & $(1.51)$ & $\mathrm{kJ} / \mathrm{kg} \mathrm{K}$ \\
\hline$c_{\mathrm{v}}$ & specific heat (constant $V$ ) & & $\mathrm{kJ} / \mathrm{kg} \mathrm{K}$ \\
\hline$D$ & downward radiation & & $\mathrm{W} / \mathrm{m}^{2}$ \\
\hline$g$ & gravitational constant & 9.81 & $\mathrm{~m} / \mathrm{s}^{2}$ \\
\hline$\Gamma$ & lapse rate & & $\mathrm{K} / \mathrm{m}$ \\
\hline$h$ & total height of atmosphere & & $\mathrm{m}$ \\
\hline$k$ & Boltzmann constant & $1.380649 \times 10^{-23}$ & $\mathrm{~J} / \mathrm{K}$ \\
\hline$m$ & molecular mass (air) & $4.82 \times 10^{-26}$ & $\mathrm{~kg}$ \\
\hline$M$ & atmospheric mass density & 10.328 & $10^{3} \mathrm{~kg} / \mathrm{m}^{2}$ \\
\hline$N_{\mathrm{A}}$ & Avogadro's constant & $6.0221476 \times 10^{23}$ & $1 / \mathrm{mol}$ \\
\hline$P$ & pressure & & $\mathrm{Pa}$ \\
\hline$P_{0}$ & surface atmospheric pressure & 1013.2 & $\mathrm{hPa}$ \\
\hline$\rho$ & mass density & & $\mathrm{kg} / \mathrm{m}^{3}$ \\
\hline$\rho^{\prime}$ & mass density & & $\mathrm{kg} / \mathrm{Km}^{2}$ \\
\hline$s$ & climate sensitivity & & K per ppm \\
\hline$S$ & solar variable $(W / 4)$ & 340 & $\mathrm{~W} / \mathrm{m}^{2}$ \\
\hline$\sigma$ & Stefan-Boltzmann constant & $5.670374419 \times 10^{-8}$ & $\mathrm{~W} / \mathrm{m}^{2} \mathrm{~K}^{4}$ \\
\hline$\sigma_{\mathrm{x}}$ & capture cross section & & $\mathrm{m}^{2}$ \\
\hline$T$ & absolute temperature & & K \\
\hline$T_{0}$ & surface temperature & (288) & K \\
\hline$T_{\mathrm{bb}}$ & black-body temperature & 254.0 & K \\
\hline$\tau$ & optical depth & & 1 \\
\hline$\tau$ & relaxation time & & s \\
\hline$U$ & upward radiation & & $\mathrm{W} / \mathrm{m}^{2}$ \\
\hline$V$ & volume & & $\mathrm{m}^{3}$ \\
\hline$w$ & radiative forcing & & $\mathrm{W} / \mathrm{m}^{2}$ per $\mathrm{ppm}$ \\
\hline$W$ & Solar constant & 1361 & $\mathrm{~W} / \mathrm{m}^{2}$ \\
\hline$z$ & altitude & & $\mathrm{m}$ \\
\hline
\end{tabular}




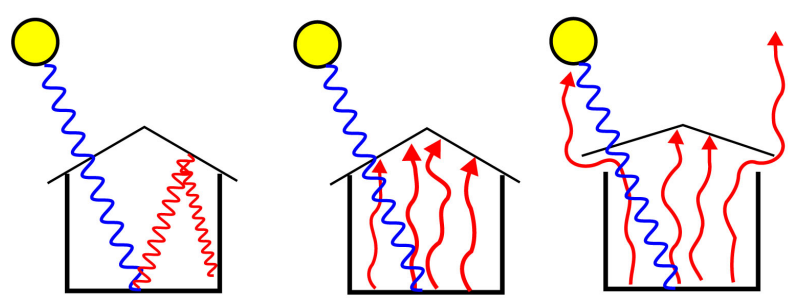

Figure 1. Left: Greenhouse effect as understood by the public at large. Short-wavelength radiation passes through the glass. Long-wavelength radiation is blocked by the glass and thus energy is trapped in the greenhouse. Middle: A greenhouse heats up by blocking hot air, heated by solar radiation, from rising. Right: Opening the roof windows would continue the greenhouse effect of the left picture, but lets the hot air trapped in the middle picture go out and cool down the greenhouse.

and oxygen using the energy of light. According to the principle of Le Chatelier, if the concentration of a reactant on the left of the reaction is increased, the reaction tends to go to the right side. $\mathrm{CO}_{2}$ is thus an effective fertilizer in plant growth, and farmers know it. Now, exactly the same reasoning upholds in the entire "greenhouse" we call planet Earth. In fact, the planet has become much greener in the last decades, as satellite measurements have shown [4]. It may also have been helped by the increased temperature, because most chemical processes in nature are thermally activated, so called Arrhenius law. Moreover, plants become more resistant to drought in a $\mathrm{CO}_{2}$-rich environment because the exchange of a $\mathrm{CO}_{2}$ molecule for an oxygen molecule at the leaf level of the plant is accomplished with the help of evaporation of water through the stomatas. The plants can keep their stomata longer closed to avoid dehydration while still flourishing.

Finally, it has to be pointed out that Knut Ångström, one of the first-if not the first-to mention the heating effect of the atmosphere and specifically the carbon dioxide in it, dismissed the greenhouse effect: "the total absorption [of Earth's radiation] is very little dependent on the changes in the atmospheric carbon dioxide content, as long as it is not smaller than 0.2 of the existing value" [5]. In other words, the greenhouse effect-if caused by radiation balance-is saturated and only starts dropping if the $\mathrm{CO}_{2}$ concentration drops below $20 \%$ of the value at the times of Angström, which would possibly be 60 ppm. Increasing the concentration above these levels has no effect whatsoever on the temperature.

\subsection{Henry's Law}

The correlation between temperature and $\left[\mathrm{CO}_{2}\right]$ is readily explained by another phenomenon, called Henry's Law: The capacity of liquids to hold gases in solution is depending on temperature. When oceans heat up, the capacity decreases and the oceans thus release $\mathrm{CO}_{2}$ (and other gases) into the atmosphere. When we quantitatively analyze this phenomenon, we see that it perfectly fits the observations, without the need of any feedback [1]. We thus now have an alternative hypothesis for the explanation of the observations presented by Al Gore. 
The greenhouse effect can be as good as rejected and Henry's Law stays firmly standing. We concluded that the effect of anthropogenic $\mathrm{CO}_{2}$ on the climate is negligible and the effect of the ocean temperature on atmospheric $\left[\mathrm{CO}_{2}\right]$ is exactly, both sign and magnitude, equal to that as expected on basis of Henry's Law [1].

\subsection{Contemporary Correlation}

However, remains the possible correlation of contemporary temperatures and $\left[\mathrm{CO}_{2}\right]$, which by some is used as an argument for $\mathrm{CO}_{2}$-caused global warming. Allegedly, both temperature and $\left[\mathrm{CO}_{2}\right]$ are rising synchronously, in lockstep. Figure 2 shows the data of Ref. [6], after careful adjusting of the scales $\left(\left[\mathrm{CO}_{2}\right]\right.$ and $T$ are in different domains, so we can adjust scales freely). The $\mathrm{CO}_{2}$ data are the monthly Mauna Loa data from which the seasonal cycle was removed in order to only reveal the trend. The temperature data are offset from long term averages (and thus also any seasonal periodicity removed) and averaged over 12 months to remove some noise.

We see that the correlation is quite good. However, we have to be careful. Because scales can be freely adjusted, basically there only exist two possibilities of outcome, just like stock market charts: They are either going up (from bottom left to top right) or down (from top left to bottom right) and a correlation between any two is guaranteed! (Either positive correlation or negative correlation). Bearing this in mind, a seeming correlation can turn out to be meaningless. We need to further analyze the data.

Correlations are best shown in correlation plots; instead of both shown as a time series, they are better shown as one vs. the other; if they are correlated, a straight line should result. Figure 2(b) shows a correlation plot of the same data as used for Figure 2(a) (but no averaging). We see that there is an apparent correlation between the two datasets and we can fit a line to them to find the coefficient. The value is $10.2 \mathrm{mK} / \mathrm{ppm}$. (See Table 2 for a summary of all data sets and models described here).

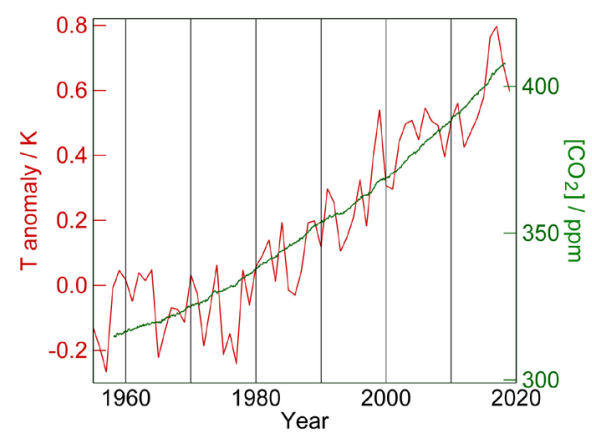

(a)

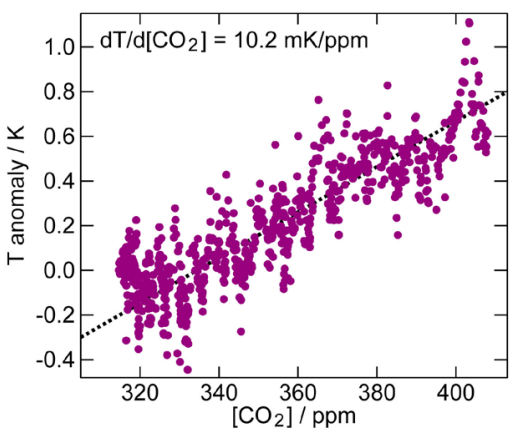

(b)

Figure 2. (a) Correlation between global temperature (red) and $\mathrm{CO}_{2}$ concentration (green) since 1958. The correlation is quite good; (b) Correlation plot of same data. In this plot monthly data are used for both $\left[\mathrm{CO}_{2}\right]$ and $T$, with $\left[\mathrm{CO}_{2}\right]$ corrected for seasonal cycles. From Ref. [6]. 
Table 2. Summary of experimental data correlation and theoretical model values.

\begin{tabular}{cc}
\hline Data/Model & Value \\
\hline Ice core data $(600 \mathrm{ka})$ & $95 \mathrm{mK} / \mathrm{ppm}[1]$ \\
Contemporary data $(60 \mathrm{a})$ & $10.2 \mathrm{mK} / \mathrm{ppm}[6]$ \\
Geological data $(650 \mathrm{Ma})$ & $-0.43 \mathrm{mK} / \mathrm{ppm}$ (this work) \\
Holocene data (11 ka) & $-43 \mathrm{mK} / \mathrm{ppm}$ (this work) \\
Greenhouse effect & $1.4 \mathrm{mK} / \mathrm{ppm}[1]$ \\
Greenhouse effect & $3.3 \mathrm{mK} / \mathrm{ppm}$ (this work) \\
Henry's Law & $100 \mathrm{mK} / \mathrm{ppm}^{*}[1]$ \\
\hline
\end{tabular}

*: Henry's Law is $10 \mathrm{ppm} / \mathrm{K}[1]$.

This experimental value of $10.2 \mathrm{mK} / \mathrm{ppm}$ is highly interesting. It is neither close to the value expected for the greenhouse effect $(1.4 \mathrm{mK} / \mathrm{ppm})$, nor close to the value of Henry's Law (100 $\mathrm{mK} / \mathrm{ppm})$. Even stranger, it is also not close to the value of the $600-\mathrm{ka}$ ice core data $(95 \mathrm{mK} / \mathrm{ppm})$.

A missing response might be explained in a relaxation model. After all, induced changes take time to materialize; the system needs time to settle to the new equilibrium value. However, too big responses compared to the model are not possible. A more likely cause for the divergence between model and data is that the correlation is merely coincidental [2]. In a Henry's-Law (HL) analysis, the $\mathrm{CO}_{2}$ has no effect on the temperature, but a concurrent temperature rise is merely a coincidence. That is, because the $\left[\mathrm{CO}_{2}\right]$ rise in contemporary data is possibly of anthropogenic origin and not (much) caused by the temperature rise. In the HL framework, the ca. 0.8 degree temperature rise has contributed a meager $8 \mathrm{ppm}$ to the $\mathrm{CO}_{2}$ in the atmosphere. The rest might be coming from anthropogenic sources, or from nature itself.

If, on the other hand, we want to attribute the temperature rise to $\mathrm{CO}_{2}$, we must build-in a delay, since most of the effect of the alleged greenhouse effect has apparently not occurred, yet. Using the value of $95 \mathrm{mK} / \mathrm{ppm}$ (Table 2), the $80 \mathrm{ppm}$ of Figure 2(b) should have produced (will produce?) a staggering 7.6 degree temperature rise. A meager 0.8 degrees is observed after 60 years (13 $\mathrm{mK} / \mathrm{a}$. Figure 2). From these data we can deduce a virtual relaxation time $\tau$, namely a relaxation time of about half a millennium. We can thus expect a temperature rise of some tenths of a degree per decade in the coming centuries. This causes a problem. Having set out to explain things in known physical laws, we have no idea what physical process might be the origin of this relaxation. The radiation balance of the atmosphere has a relaxation time of about a month and a half, as evidenced by the 30 -day belay between shortest/longest day and coldest/warmest day [1]. No millennium-scale relaxation mechanisms are easily identifiable in the atmosphere, where the GHE resides. We can even exclude that the oceans act as thermal sinks for the heat generated in the atmosphere, because then the effect in the atmosphere would initially be even larger than theoretically 
predicted, in an effect called overshoot. We thus conclude that, upon scrutiny, the (alleged) greenhouse effect also has to be rejected as a hypothesis to explain contemporary data. Can the data be explained by Henry's Law, instead?

The observed correlation is $10 \mathrm{mK} / \mathrm{ppm}$, or conversely $100 \mathrm{ppm} / \mathrm{K}$. That is a factor 10 too big for Henry's Law, and relaxation processes can only make the effect smaller. We thus exclude Henry's Law as an explanation for the contemporary steady $\left[\mathrm{CO}_{2}\right]$ rise in the atmosphere, it is not caused by the steady rise in temperature.

In a recent publication, however, we analyzed quasi-periodic signals of temperature and $\left[\mathrm{CO}_{2}\right]$. More precisely, the correlation of ocean surface temperature and $\mathrm{CO}_{2}$ concentration in the atmosphere, and we found that these signals are perfectly explained by Henry's Law, with a relaxation time (slow warming up and outgassing of oceans) commensurate the data. We have thus shown that empirically the greenhouse effect can be rejected-it is rather insignificant-and there is a very plausible explanation for all the observed data. Thus limiting the magnitude of the greenhouse effect, what is still missing is to find a theoretical estimate of this greenhouse effect. This is what this work is trying to do. It will do that analytically, to keep a full eye on what is going on.

\section{A Radiative Greenhouse Model}

\subsection{Absorption in the Atmosphere}

An intrinsic assumption we make here is that all incoming energy comes from radiation from the Sun. Heat coming from the Earth itself, from below the crust, is too small (about $50 \mathrm{~mW} / \mathrm{m}^{2}$; estimation from the authors) to be significant. Moreover, all heat must be dissipated to the universe by radiation only. Things as evaporation of hot molecules from the top of the atmosphere is too insignificant. This is a rather trivial assumption and will therefore not be further justified. Perhaps more questionable is the assumption that the atmosphere is a well mixed chamber, meaning that all gases occur in the same ratios everywhere.

The theoretical greenhouse effect is governed by optical absorption and emission processes in the atmosphere. As such, the Beer-Lambert rule of absorption plays an important role. We start with the important definitions of phenomena and parameters.

If every molecule has an absorption cross section of $\sigma_{\mathrm{x}}$ (unit: $\mathrm{m}^{2}$ ) independent of the light intensity, and the concentration of the molecule is $c$ (unit: $1 / \mathrm{m}^{3}$ ), the attenuation of a light beam with intensity $J\left(\right.$ unit: $\left.\mathrm{W} / \mathrm{m}^{2}\right)$ due to absorption is

$$
\frac{\mathrm{d} J(z)}{\mathrm{d} z}=-\sigma_{\mathrm{x}} c J(z),
$$

with $z$ the space coordinate along the path of radiation. We can see $\sigma_{\mathrm{x}}$ as the probability a photon is captured when it passes nearby a molecule; if it passes through the capture cross section area it is captured, otherwise it just flies by (larger cross-section gives larger probability). Important to note for further analysis, $\sigma_{\mathrm{x}}$ is a property of the specific molecule, and not depending on the 
concentration of this molecule. More useful is to define an average $\sigma_{\mathrm{x}}$ of an average molecule in the atmosphere that will then be a weighted average of the cross sections of the individual constituents of the atmosphere, useful for calculations when the composition of the atmosphere changes.

Now, in normal laboratory conditions, the energy of the absorbed photons is dissipated into an infinite heat bath; i.e. no photons are reemitted! Once the energy is absorbed, it stays in the absorbant. This is the analysis shown here, which has a solution that is the Beer-Lambert law, that for a homogeneous medium is:

$$
J(z)=J_{0} \exp \left(-\sigma_{\mathrm{x}} c z\right) .
$$

To describe the opacity of matter, often an absorption coefficient,

$$
\alpha \equiv \sigma_{\mathrm{x}} c,
$$

is used (unit: $1 / \mathrm{m}$ ), which then depends on the type and concentration of the absorber. Also sometimes the concept of "optical depth" $(\tau)$, or "absorbance" when working in 10-base instead of e-base, is introduced, which is the logarithm of the ratio of incoming light and outgoing light, and thus equal to the product of absorption coefficient $(\alpha)$ and optical path $(z): \tau \equiv \ln \left(J_{0} / J(z)\right)=\sigma_{x} c z$. In the more-correct inhomogeneous approach of the atmosphere, the system can be solved by the alternative form of the first equation, with $c(z)$ a function of $Z$, and then gives a solution with the optical depth linearly proportional to the total amount of absorbing matter: Optical depth $(\tau)$ is the natural logarithm of opacity $J_{0} / J(h)$ (where absorbance is its 10-base-logarithm):

$$
\tau \equiv \ln \left(\frac{J_{0}}{J(h)}\right)=\sigma_{\mathrm{x}} C
$$

with $C$ the total density per area (unit: $1 / \mathrm{m}^{2}$ ) of the absorbant, $C=\int_{0}^{h} c(z) \mathrm{d} z$, with $h$ the length of the total path in the absorbing body (viz. height of atmosphere). The transmitted intensity $J(h)$ is exponentially dependent on the optical depth; doubling the total amount of absorbant in the atmosphere doubles the optical depth and the transmitted intensity $b$ quadratically drops, for instance from $10 \%$ to $10 \%^{2}=1 \%$. In other words, the transmittance of the body of gas is equal to

$$
b \equiv \frac{J(h)}{J_{0}}=\exp \left(-\sigma_{\mathrm{x}} C\right) .
$$

The greenhouse effect is often erroneously presented as being caused by the fact that $\sigma_{\mathrm{x}}$ (and thus also $\alpha, \tau$ and absorbance) depends on the wavelength. That visible sunlight can reach the surface and infrared terrestrial light cannot easily escape through the atmosphere. This schematic presentation is wrong, however, as we will discuss. It does not matter how and where the solar radiation is absorbed or terrestrial IR radiation is absorbed and reemitted in the atmosphere, if sunlight reaches the surface or not, etc. The only thing that matters is the amount of radiation received by Earth (that is, the amount that is not 
reflected directly back into space) and amount of IR radiation emitted.

We must start, however, by stating that it is exactly here where the greenhouse theory diverges from classical Beer-Lambert absorption theory presented above, because in the real atmosphere, the energy absorbed cannot sink into a heat reservoir, it must be reemitted somehow by radiation in the classic greenhouse theory; the greenhouse effect is an approach of describing the atmosphere exclusively by radiation balances. This makes the Beer-Lambert Equation (possibly) no longer valid. It is this that we will calculate here to find an estimate for the greenhouse effect. We will try to calculate the radiative forcing and temperature effect of changing the composition of the atmosphere, assuming only radiation redistributes heat.

Since the absorption coefficient depends on capture cross-sections as well as concentrations, pumping $\mathrm{CO}_{2}$ in the atmosphere might increase heat absorption in the atmosphere and might thus heat it up. However, at first sight, the effect is probably minimal, because nearly all infrared light is already absorbed; at the top of the atmosphere, according to the Beer-Lambert Equation (Equation (2)): $J(h) \approx 0$. We thus do not expect much effect from adding $\mathrm{CO}_{2}$ to the atmosphere as long as there are other channels open for emission. Imagine: if $99 \%$ of the light possibly absorbed is already absorbed $\left(\right.$ say, $\left.350 \mathrm{~W} / \mathrm{m}^{2}\right)$, doubling $\mathrm{CO}_{2}$ in the atmosphere will not double the absorption (to $700 \mathrm{~W} / \mathrm{m}^{2}$ ), but just add something close to $1 \%$ to it $\left(3.5 \mathrm{~W} / \mathrm{m}^{2}\right)$, as Equation (2) tells us. We call this the "forcing" of the atmosphere. How much does this forcing change the temperature of the surface? If the wavelengths of $\mathrm{CO}_{2}$ are the only channels at which radiation can reach space, exactly because $J(h) \approx 0$, the effect may still be dramatic, as caused by these non-Beer-Lambert effects.

We now have all the ingredients to calculate these effects. We must first calculate the equilibrium temperature of our planet without an atmosphere, which is quite easy, if not controversial as well. The surface of the planet with a (Bond) albedo of a $(0.306 ; 30.6 \%$ of light is reflected, $69.4 \%$ is absorbed), receives $S$ solar radiation $\left(\mathrm{W} / \mathrm{m}^{2}\right)$, where $S$ can be found from the solar constant $W\left(1361 \mathrm{~W} / \mathrm{m}^{2}\right)$ which is the solar radiation density in space [7]. On a globe, rotating with angle $\phi$, at a latitude $\theta$, a radiation is received per area equal to

$$
W_{\theta}=\frac{\int_{-\pi / 2}^{\pi / 2} W \cos \phi \cos \theta \mathrm{d} \phi}{\int_{-\pi}^{\pi} \mathrm{d} \phi}=\frac{W}{\pi} \cos \theta
$$

Now, the average radiation received per square meter on this planet is then found by weighted averaging (weight proportional to $\cos \theta$ ) over the entire sphere,

$$
\begin{gathered}
S=\frac{\int_{-\pi / 2}^{\pi / 2}(W / \pi) \cos \theta(2 \pi R)(R \cos \theta) \mathrm{d} \theta}{\int_{-\pi / 2}^{\pi / 2}(2 \pi R)(R \cos \theta) \mathrm{d} \theta} \\
=\frac{\pi R^{2} W}{4 \pi R^{2}}=\frac{W}{4},
\end{gathered}
$$

the amount of radiation passing through a disk with radius $R$ divided by the 
surface area of the planet with same radius. Thus, $S=340 \mathrm{~W} / \mathrm{m}^{2}$. A fraction equal to the albedo or "whiteness" ( $a=0.306$ ) is directly reflected back into the universe and the rest, $(1-a) S=236 \mathrm{~W} / \mathrm{m}^{2}$, is absorbed by the surface. The surface emits thermal radiation $G$ that depends on temperature, according to Stefan and Boltzmann:

$$
G=\sigma T_{0}^{4},
$$

with $\sigma$ the constant of Stefan-Boltzmann (see the Table 1 for values of parameters). Note that there is no albedo in this equation, implying that for emission the Earth is a black body (albedo $a=0$; emissivity $\varepsilon \equiv(1-a)=1$, which is of high importance. The reason why will be explained later). Kirchoff's law of thermal radiation states that "For an arbitrary body emitting and absorbing thermal radiation in thermodynamic equilibrium, the emissivity is equal to the absorptivity". When we set the absorption $(1-a) S$ equal to the emission $G$, gives us the temperature of the planet without atmosphere, the so-called black-body temperature:

$$
T_{\mathrm{bb}}=\sqrt[4]{\frac{(1-a) S}{\sigma}}=254.0 \mathrm{~K}
$$

We must remark at this moment that this is the temperature of Earth as seen from outer space. Irrespective of any greenhouse or other effect. If we, from outer space, point a radiometer at the planet it will have a temperature signature of $254.0 \mathrm{~K}$. (If we also include the visible light that is reflected (aS), then the total radiation power is equal to that of an black sphere with temperature $T=\sqrt[4]{S / \sigma}=278.3 \mathrm{~K}$ ). The greenhouse effect does not change the apparent temperature of the planet as seen from outer space, but only that of a hidden layer (e.g., the solid surface). Radiation into space effectively comes from the atmosphere at an altitude where the temperature is $254.0 \mathrm{~K}$, which is in the troposphere at about $6 \mathrm{~km}$ height.

The controversy in this is effectively assuming the Earth is a flat surface, or that the received heat is immediately equalized along the planet, with the same temperature everywhere. If, on the other hand, we were to calculate the local temperature $T_{\theta}$ on basis of a radiation balance at every point, $(1-a) W_{\theta}=\sigma T_{\theta}^{4}$, disallowing thermodynamic processes of heat redistribution altogether, we would find an average temperature of

$$
T_{\mathrm{bb}, \text { sphere }}=\frac{\int_{-\pi / 2}^{\pi / 2} \sqrt[4]{W(1-a) \cos \theta / \sigma \pi}(2 \pi R)(R \cos \theta) \mathrm{d} \theta}{4 \pi R^{2}}=251.2 \mathrm{~K} .
$$

slightly lower. We will, however, continue with the "flat-earth" value of $T_{\mathrm{bb}}=$ $254.0 \mathrm{~K}$, and a flat-earth analysis in general, because we want to study the greenhouse phenomenon. Bear in mind that the real spherical greenhouse effect is always a little bigger than the ones calculated, by about $2.8 \mathrm{~K}$.

Another controversy exists about what the real temperature is on Earth. Some mention 287.0 K [6]. Other sources mention different global temperatures. For instance Ref. [8] that give $288.2 \mathrm{~K}$. Since we are mostly interested in 
relative effects, either value will do; we'll use $288 \mathrm{~K}$. We just mention this one here to show that there is not even a consensus on what the temperature of the planet is.

We now first analyze the atmosphere in a two-box pseudo-Beer-Lambert model: The radiation $G$ from the surface is transmitted with a factor $b$ directly into outer space, according to the Beer-Lambert law shown above (Equation (5)). The absorbed radiation is re-emitted, the atmosphere considered being a single body, half goes into space and half goes back to Earth. This downward radiation $(1-b) G / 2$ is what is added to the radiation received from the Sun, $(1-a) S$, and this is what heats up the surface above its atmosphereless temperature $T_{\mathrm{bb}}$. We get the situation as in Figure 3. When we analyze the top of the atmosphere and once again set equilibrium conditions, we get

$$
S=a S+b G+\frac{1-b}{2} G
$$

which gives a solution according to Equation (9) of

$$
T_{0}=\sqrt[4]{\frac{2(1-a)}{\sigma(1+b)} S} .
$$

A similar result we would get when analyzing the equilibrium at the surface. Substituting the real temperature $\left(T_{0}=288 \mathrm{~K}\right)$, the solar constant $\left(S=340 \mathrm{~W} / \mathrm{m}^{2}\right)$ and the albedo ( $a=0.306$ ), we find $b=0.210$. Now if we assume all absorption is due to $\mathrm{CO}_{2}$ (or analyzing the effect of the entire atmosphere), doubling its density $C$ will square this constant: $b^{\prime}=b^{2}=0.044$, according to Beer-Lambert (Equation (5)). Substituting this value into our two-box model Equation (13), gives us $T_{0}=298.8 \mathrm{~K}$, or a climate sensitivity of $\mathrm{CO}_{2}$ of $\Delta T_{0} / \Delta\left[\mathrm{CO}_{2}\right]=$ $(10.8 \mathrm{~K}) /(350 \mathrm{ppm})=30.9 \mathrm{mK} / \mathrm{ppm}$. The real effect must be much less, if existing at all, and our model too simplistic. The problem is that the atmosphere is not a single layer.

We now proceed to simulate the atmosphere as a multilayer body. Each layer receives radiation from below as well as radiation from above (light that was absorbed and re-emitted). The result is that more heat can escape from the bottom of the atmosphere than from the top of the atmosphere, as the following calculation will show.

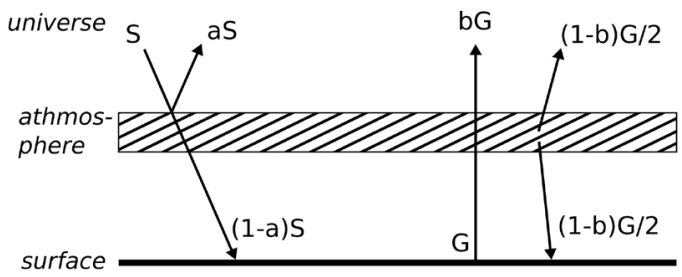

Figure 3. Two-box model of Earth/atmosphere. $S$ is the radiation which is received by the planet per square meter. A fraction $a$ of it is reflected back into space and $(1-a)$ absorbed. The surface radiates heat according to Stefan-Boltzmann (Equation (9)), a fraction $b$ of which directly reaches space (according to Beer-Lambert Equation (2)). The rest is absorbed and re-emitted. 
For an infinitesimal thin layer at height $z$ we have radiation $U$ coming in from below going up and radiation $D$ coming from above going down, as shown in Figure 4(a). The layer absorbs parts of them according to Beer-Lambert (Equations (1) and (3)). Then, half of that absorbed heat is re-emitted downwards and half upwards in this analysis, because the layer itself is considered a uniform single body.

Looking at Figure 4, this results in a coupled differential equation

$$
\begin{aligned}
& \frac{\mathrm{d} U}{\mathrm{~d} z}=\frac{\alpha(D-U)}{2}, \\
& \frac{\mathrm{d} D}{\mathrm{~d} z}=\frac{\alpha(D-U)}{2} .
\end{aligned}
$$

With boundary conditions that the upwards radiation at ground level is equal to the thermal emission form the surface, $U(0)=G$, and the downwards radiation at the top of the atmosphere is zero, $D(h)=0$, the result is

$$
\begin{aligned}
& U(z)=\left(1-\frac{\alpha z}{\alpha h+2}\right) G, \\
& D(z)=\frac{\alpha(h-z)}{\alpha h+2} G .
\end{aligned}
$$

Remarkably, these functions are linear (see Figure 4(b)), clearly deviating from Beer-Lambert (exponential) behavior. Compare the above function for $U(z)$ with the Beer-Lambert behavior of $J(z)$ of Equation (2). Moreover, only in lowconcentration conditions (small $\alpha h$ ) can $\alpha$ and $\sigma_{\mathrm{x}}$ be extracted through the optical depth, the logarithm of intensity $U(h)$ (Equation (4)), in any case losing a factor 2 , caused by the absence of a heat bath.

At the top and bottom of the atmosphere comes out, respectively

$$
\begin{aligned}
& U(h)=\frac{1}{1+\alpha h / 2} G, \\
& D(0)=\frac{\alpha h / 2}{1+\alpha h / 2} G .
\end{aligned}
$$

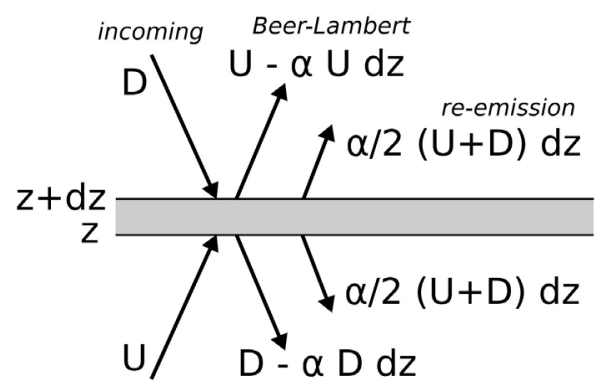

(a)

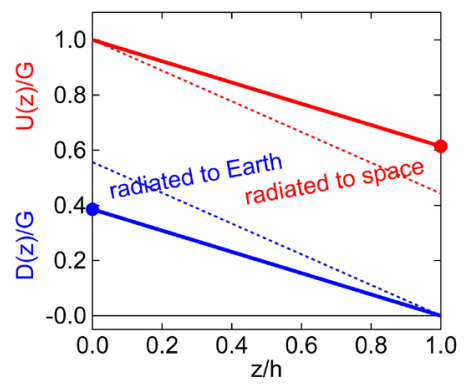

(b)

Figure 4. (a) A single layer in a multi-layer model of Earth/atmosphere. It shows the radiation flux coming in from below $(U)$ and above $(D)$ and the absorption and re-emission processes; (b) Normalized upward $(U(z)$, red) and downward $(D(z)$, blue) radiation as a function of altitude $(z)$ scaled to total height of atmosphere $(h)$. Solid line is current surface temperature, dashed line is for doubling of $\left[\mathrm{CO}_{2}\right]$. 
Demanding equilibrium at the top of the atmosphere, and substituting $G=U(0)$ $=\sigma T_{0}^{4}$ :

$$
S=a S+U(h)
$$

gives

$$
\alpha h / 2=\frac{\sigma T_{0}^{4}}{(1-a) S}-1
$$

Substituting the current temperature $T=15.0^{\circ} \mathrm{C}$ and albedo gives $\alpha h / 2=0.6557$.

Note that the radiative forcing is thus not logarithmically depending on the concentration of the absorbant, as some claim [9]. Radiative forcing can be calculated as

$$
R F \equiv D(0)=\frac{\alpha h}{2}(1-a) S,
$$

and is thus linearly proportional on concentration $c$, because $\alpha=c \sigma_{\mathrm{x}}$. Per ppm it is constant.

In this analysis we assume that radiation balances are determining the atmosphere. The temperature can then be found from the radiation intensity by the inverse function of Stefan-Boltzmann. With the emission $\alpha(U+D) \mathrm{d} z$ linearly depending on height, this results in a fourth-root curve of height, with $T=288 \mathrm{~K}$ at the surface $(z=0)$ and about $210 \mathrm{~K}\left(-60^{\circ} \mathrm{C}\right)$ at the top of the atmosphere. This is obviously incorrect.

In the extreme, in this model attributing all greenhouse effect to $\mathrm{CO}_{2}$, doubling $c=\left[\mathrm{CO}_{2}\right]$ will double $\alpha$ and that will result in a temperature of $T_{0}=313.2 \mathrm{~K}$, or in other words, a sensitivity of $\Delta T_{0} / \Delta\left[\mathrm{CO}_{2}\right]=(25.1 \mathrm{~K}) /(350 \mathrm{ppm})=71.7$ $\mathrm{mK} / \mathrm{ppm}$. For further reference: The value of $\alpha h / 2=0.6557$ means that in the current situation $61.4 \%$ of the radiation $G$ coming from the surface leaves from the top of the atmosphere and the rest, $38.6 \%$, from the bottom of the atmosphere (according to Equation (16)). Note also that a part $\exp (-\alpha h)=$ $28.5 \%$ is never absorbed by the atmosphere. The rest, $71.5 \%$, is absorbed and re-emitted, somewhere, at least once.

\subsection{A Non-Uniform Atmosphere}

In the analysis above, it was assumed that the atmosphere was a homogeneous well-mixed closed box of height $h$ with constant properties everywhere: pressure, concentrations, absorption constants, etc. (Except for a radiation gradient of Figure 4(b)). A real atmosphere, on the other hand, has no upper boundary and is contained by gravity on one side, making it ever thinner with height. We can calculate the properties of such a system. We start with the ideal gas law,

$$
P V=N k T,
$$

with $P$ pressure, $V$ volume, $N$ the number of molecules, $k$ Boltzmann's constant, and $T$ the absolute temperature. The density of the gas can be defined as 


$$
\rho=\frac{N m}{V}
$$

with $m$ the mass of a single molecule of air $\left(29 \mathrm{~g} / \mathrm{mol}\right.$, thus $\left.m=4.82 \times 10^{-26} \mathrm{~kg}\right)$. When we substitute this into the ideal gas law we get

$$
\rho=\frac{P m}{k T} .
$$

Now, a layer of the atmosphere with thickness $\mathrm{d} z$ is in equilibrium with two forces acting upon it: a gravitational pull and differential-pressure force, respectively given by (see Figure 5)

$$
\begin{aligned}
& F_{\text {down }}=g \rho A \mathrm{~d} z, \\
& F_{\text {up }}=-A \mathrm{~d} P,
\end{aligned}
$$

with $A$ the area of the layer, and $g$ the gravitational constant $\left(9.81 \mathrm{~m} / \mathrm{s}^{2}\right)$. Setting them equal means

$$
\frac{\mathrm{d} P}{\mathrm{~d} z}=-g \rho
$$

Substituting the ideal-gas-law density of Equation (22), moreover assuming a constant temperature (sic), we find the textbook so-called barometric formula:

$$
P(z)=P_{0} \exp \left(-\frac{m g}{k T} z\right) .
$$

In which $P_{0}$ can be found by integration of the density Equation (Equation (22)) using the above equation for $P(z)$ and mounts up to summing all the mass $M$ per square meter hanging above us.

$$
M=\int_{0}^{\infty} \rho(z) \mathrm{d} z=\frac{P_{0}}{g} .
$$

Assuming $g$ not depending on altitude, $P_{0}=g M$. It has to be noted that this constant is independent of temperature (or gradient) and pressure gradient in the atmosphere, and only depends on its mass; it is simply the weight of the atmosphere. Also, sometimes arguments are heard that the temperature is a result of the pressure [10]. But that is misinterpreting the ideal-gas law. This law merely states an equality and does not imply cause and effect.

In the above, a constant temperature was assumed, but in reality the temperature depends on height, as is not difficult to show: We can find the lapse-rate, $\Gamma \equiv \mathrm{d} T / \mathrm{d} z$, when we realize that no energy $(E)$ is added to the atmosphere in steady state and thus it is adiabatic, defined as

$$
\mathrm{d} E=c_{\mathrm{p}} P \mathrm{~d} V+c_{\mathrm{v}} V \mathrm{~d} P=0,
$$

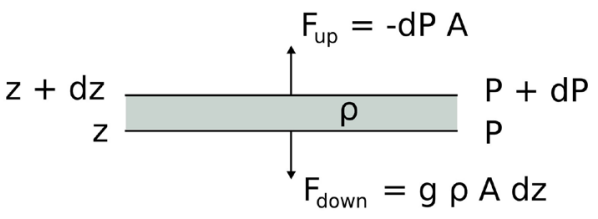

Figure 5. Forces acting upon a layer of the atmosphere. 
or

$$
P \mathrm{~d} V=-\left(c_{\mathrm{v}} / c_{\mathrm{p}}\right) V \mathrm{~d} P
$$

with $c_{\mathrm{p}}$ and $c_{\mathrm{v}}$ the specific heat ( $\mathrm{d} Q / \mathrm{d} T$ per mass. Unit: $\mathrm{J} / \mathrm{K} \mathrm{kg}$ ) at constant pressure and constant volume, respectively. It follows the first law of thermodynamics that energy added to the system is either internal heat or work done:

$$
\Delta E=Q-W
$$

with $E$ he total energy, $Q$ the internal heat and $W$ the work being done. For adiabatic processes $\Delta E=0$. Changes in internal heat are given by the mass times the specific heat (at constant volume) times the change of temperature

$$
\mathrm{d} Q=N m c_{\mathrm{v}} \mathrm{d} T
$$

and the work done is

$$
W=P \mathrm{~d} V,
$$

With $\Delta E=0$, and substituting the adiabatic definition, and dividing both sides by $\mathrm{d} z$ we get

$$
N m c_{\mathrm{p}} \frac{\mathrm{d} T}{\mathrm{~d} z}=V \frac{\mathrm{d} P}{\mathrm{~d} z} .
$$

With our pressure gradient found before (Equation (24)), and knowing $\rho=N m / V$, we find our lapse rate:

$$
\Gamma \equiv \frac{\mathrm{d} T}{\mathrm{~d} z}=-\frac{g}{c_{\mathrm{p}}} .
$$

We could have gotten this result much faster if we had started from the specific energy (energy per $\mathrm{kg}$ ) equation of a package of air, $e=c_{\mathrm{p}} T+g z$, and realized that in equilibrium $\mathrm{d} E$ and $\mathrm{d} e$ are zero (all packages with same energy), hence the equation of the lapse rate above. The specific heat of (dry) air is 1.006 $\mathrm{kJ} / \mathrm{kg} \mathrm{K}[11]$, so $\Gamma_{\text {dry }}=-9.75 \mathrm{~K} / \mathrm{km}$. The impact of carbon dioxide on this lapse rate is minimal, because the mole fraction, and partial pressure, of $\mathrm{CO}_{2}$ is negligible. Yet, if we were to replace the atmosphere by one fully made up of carbon dioxide, the specific heat would be $0.844 \mathrm{~kJ} / \mathrm{kg} \mathrm{K}$ and the lapse rate $-11.6 \mathrm{~K} / \mathrm{km}$. More relevant is the specific heat of moist air. It is much higher,

$$
c_{\mathrm{p}}=1.001+1.84 x \mathrm{~kJ} / \mathrm{kg} \cdot \mathrm{K},
$$

with $x$ the humidity ratio $(\mathrm{kg} / \mathrm{kg})$, and this makes the lapse rate in a humid atmosphere significantly lower (with $x$ equal to $0.277, c_{\mathrm{p}}=1.51 \mathrm{~kJ} / \mathrm{kg} \cdot \mathrm{K}$ ). An international standard atmosphere, based on typical humidity conditions, has a lapse rate of [12]

$$
\Gamma_{\text {standard }}=-6.49 \mathrm{~K} / \mathrm{km} \text {. }
$$

We notice this when we go into the mountains. It gets a degree colder for every 150 meters, or so. Table 3 summarizes the parameters.

We can now go back to the determination of the pressure curve. We had assumed that the temperature was constant, and arrived at an exponentially 
Table 3. Properties of relevant gases. Source: Ref. [13]. (The specific heat of standard air was reverse-engineered form the lapse rate).

\begin{tabular}{cccc}
\hline Gas & $\begin{array}{r}\text { Specific heat } \\
(\mathrm{kJ} / \mathrm{kg} \mathrm{K})\end{array}$ & $\begin{array}{r}\text { Lapse rate } \\
(\mathrm{K} / \mathrm{km})\end{array}$ & $\begin{array}{c}\text { Molecular mass } m \\
(\mathrm{~kg})\end{array}$ \\
\hline Air (dry) & 1.006 & -9.75 & \\
Air (standard) & 1.51 & -6.49 & $4.82 \times 10^{-26}$ \\
$\mathrm{CO}_{2}$ & 0.844 & -11.6 & $7.30 \times 10^{-26}$ \\
\hline
\end{tabular}

decaying pressure (Equation (25)). Now we have a real temperature curve; integrating the lapse rate of Equation (33) we namely get

$$
T(z)=T_{0}-\frac{g}{c_{\mathrm{p}}} z
$$

Combining this with the hydraulic equilibrium equation, of the pressure gradient of Equation (24) and the ideal gas law, Equation (22), results in a first order differential equation

$$
\frac{\mathrm{d} P(z)}{\mathrm{d} z}=-\frac{g m P(z)}{k\left(T_{0}-g z / c_{\mathrm{p}}\right)},
$$

that has the solution

$$
P(z)=P_{0}\left(1-\frac{g}{c_{\mathrm{p}} T_{0}} z\right)^{m c_{\mathrm{p}} / k},
$$

and through Equation (22), we get a density curve equal to

$$
\rho(z)=\frac{m P_{0}}{k T_{0}}\left(1-\frac{g}{c_{\mathrm{p}} T_{0}} z\right)^{m c_{\mathrm{p}} / k-1} .
$$

(Standard air has a zero-altitude density of $\rho(0)=1.226 \mathrm{~kg} / \mathrm{m}^{3}$ ).

The equations are summarized in Figure 6, for which were used $m=4.82 \times$ $10^{-26} \mathrm{~kg}$ (based on $29 \mathrm{~g} / \mathrm{mol}$ ), $c_{\mathrm{p}}=1.51 \mathrm{~kJ} / \mathrm{kg} \cdot \mathrm{K}$ (derived from $\Gamma_{\text {standard }}=-6.49$ $\mathrm{K} / \mathrm{km}), \quad T_{0}=15^{\circ} \mathrm{C}(288.0 \mathrm{~K}), \quad P_{0}=1013.2 \mathrm{hPa}$ (both from Ref. [8]), $g=$ $9.81 \mathrm{~m} / \mathrm{s}^{2}$. This is the atmosphere in equilibrium (without any form of transport, by convection nor by any other means) with all gases well mixed, moreover without external heat sources. The exponent is approximately $m c_{\mathrm{p}} / k=5.27$. It seems to work quite well, rather indistinguishable from the empirical data. At least for the lower atmosphere. The equations go into weird territory for $z>c_{\mathrm{p}} T_{0} / g$ (ca. $44 \mathrm{~km}$ ) and it is obvious that the ideal-gas-law analysis must begin to fail there, since negative pressures, densities and temperatures obviously do not make sense. In reality we can see that at least up to, say, $11 \mathrm{~km}$ the analysis seems to be performing quite well, but we also bear in mind the limitations of the analysis here. We are trying to explain the greenhouse effect phenomenologically.

The total mass should be the integral of density; the integral of Equation (39) upto the altitude where the temperature is theoretically zero is 


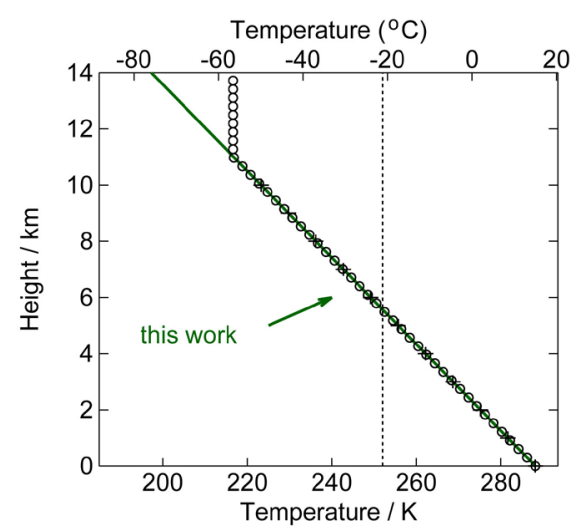

(a)

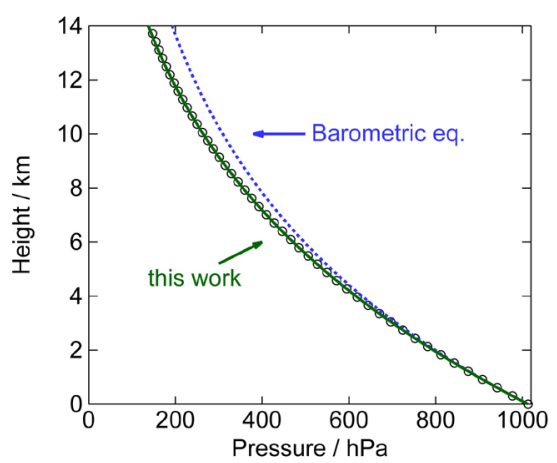

(b)

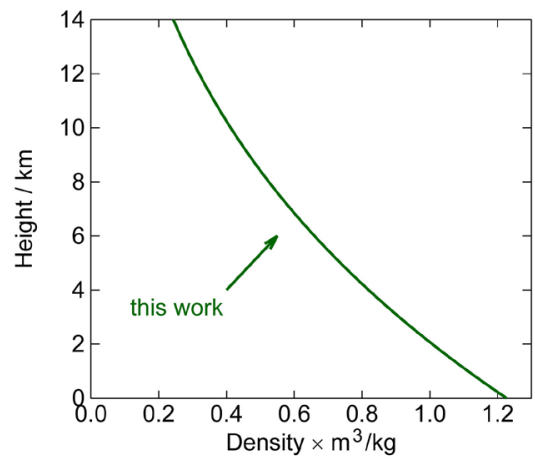

(c)

Figure 6. Adiabatic, static atmosphere without heat sources or sinks. (a) Temperature as a function of height, the slope of this linear curve is called the lapse rate and is -6.49 $\mathrm{K} / \mathrm{km}$. The black open circles and " + " signs are US standard atmosphere (Refs. [8] and [14], respectively). The green line is Equation (36). The vertical line at $254 \mathrm{~K}$ is where the outward radiation apparently is coming from; (b) Pressure as a function of height. The black dots are from Ref. [8]. The blue dashed line is the classical barometric equation, Equation (25), and the green solid line is Equation (38); (c) Density as a function of height, according to Equation (39). (Parameters used: $m=4.82 \times 10^{-26} \mathrm{~kg}, c_{\mathrm{p}}=1.51$ $\left.\mathrm{kJ} / \mathrm{kg} \cdot \mathrm{K}, \quad T_{0}=15^{\circ} \mathrm{C}, \quad P_{0}=1013.2 \mathrm{hPa}, \quad g=9.81 \mathrm{~m} / \mathrm{s}^{2}\right)$. The curves here are indistinguishable from the empirical data; the atmosphere is apparently in thermodynamic equilibrium.

$$
\int_{0}^{c_{\mathrm{p}} T_{0} / g} \rho(z) \mathrm{d} z=\frac{P_{0}}{g}=M,
$$

which is indeed equal to the result found before for the classical exponential atmosphere. We thus define the three basic functions $T(z), P(z)$ and $\rho(z)$ to be limited to $z<c_{\mathrm{p}} T_{0} / g$.

We have to make the very important observation that these curves and equations here are independent of any absorption of radiation in the atmosphere, for instance the greenhouse effect discussed earlier (Figure 4(b)). The lapse rate is not caused by radiation, but is a thermodynamic property of the atmosphere. That is, as long as there is thermodynamic equilibrium in the atmosphere, the curves are as given by the above three formulas that are determined by only the 
surface temperature $T_{0}$ and total air weight $P_{0}$, and physical properties $m$ and $c_{\mathrm{p}}$ of the gas and the gravitational constant $g$. It might, of course, be the case that the atmosphere is not in equilibrium. In that case, mass and heat can be transported through convection, diffusion, conduction and radiation. The latter two only heat (and no mass). This includes for example the effect Douglas Cotton calls "heat creep" [15], or the Connollies call "pervection" [16]. The cold upper atmosphere can absorb solar radiation and this absorbed heat is then transported to the hotter (sic) lower atmosphere that warms up by it, thereby seemingly going against the second law of thermodynamics that is often stated as "heat flows naturally from an object at a higher temperature to an object at a lower temperature, and heat doesn't flow in the opposite direction of its own accord" [17]. The second law, however, merely states that entropy increases, which it does in heat creep. We'll come back to it later. We merely state here that the atmosphere is empirically well described by a thermodynamic equilibrium.

The above equations, $T(z), P(z)$ and $\rho(z)$, have only one free parameter, $T_{0}$, all others are physical constants or are to be considered static properties, like $P_{0}$. Changes in radiation might thus change $T_{0}$. The effect of this is that an increase in $T_{0}$ shifts the temperature curve to the right, but also distorts (non-linearly) the pressure and density curves; mass is relocated away from the surface and the upper-atmospheric pressure increases.

We can now make again a radiation analysis. Substituting $\alpha=\sigma_{\mathrm{x}} c=\sigma_{\mathrm{x}} \rho(z) / m$, our coupled differential Equation (Equation (14)) becomes

$$
\begin{aligned}
& \frac{\mathrm{d} U}{\mathrm{~d} z}=\frac{\sigma_{\mathrm{x}} \rho(z)}{2 m}(D-U), \\
& \frac{\mathrm{d} D}{\mathrm{~d} z}=\frac{\sigma_{\mathrm{x}} \rho(z)}{2 m}(D-U) .
\end{aligned}
$$

(Note: The first derivatives being equal, $D$ and $U$ differ only by a constant). It leads to

$$
\begin{aligned}
& \frac{\mathrm{d}^{2} D}{\mathrm{~d} z^{2}}-\frac{1}{\rho}\left(\frac{\mathrm{d} \rho}{\mathrm{d} z}\right)\left(\frac{\mathrm{d} D}{\mathrm{~d} z}\right)=0, \\
& \frac{\mathrm{d}^{2} U}{\mathrm{~d} z^{2}}-\frac{1}{\rho}\left(\frac{\mathrm{d} \rho}{\mathrm{d} z}\right)\left(\frac{\mathrm{d} D}{\mathrm{~d} z}\right)=0 .
\end{aligned}
$$

Substituting the density function $\rho(z)$ of Equation (39), and using boundary condition $D\left(z=c_{\mathrm{p}} T_{0} / g=h\right)=0$, gives

$$
\begin{aligned}
& U(z)=D_{0}\left(1-\frac{g}{c_{\mathrm{p}} T_{0}} z\right)^{m c_{\mathrm{p}} / k}+\frac{2 g m}{\sigma_{\mathrm{x}} P_{0}} D_{0}, \\
& D(z)=D_{0}\left(1-\frac{g}{c_{\mathrm{p}} T_{0}} z\right)^{m c_{\mathrm{p}} / k} .
\end{aligned}
$$

At the boundaries $z=0$ and $z=h$ of the atmosphere the radiation densities are given by 


$$
\begin{aligned}
& U(0)=\left(1+\frac{2 g m}{\sigma_{\mathrm{x}} P_{0}}\right) D_{0}, \\
& U(h)=\frac{2 g m}{\sigma_{\mathrm{x}} P_{0}} D_{0}, \\
& D(0)=D_{0}, \\
& D(h)=0 .
\end{aligned}
$$

As before, demanding equilibrium at the top of the atmosphere requires $U(h)=$ $(1-a) S$, so

$$
D_{0}=\frac{(1-a) S \sigma_{\mathrm{x}} P_{0}}{2 g m} .
$$

The surface emits a radiation $U(0)$ upwards that is given by Stefan-Boltzmann's law, $\sigma T^{4}$, and thus we reach the final result:

$$
T=\sqrt[4]{\frac{(1-a) S}{\sigma}\left(1+\frac{\sigma_{\mathrm{x}} P_{0}}{2 g m}\right)} .
$$

Substituting the actual temperature $(288.0 \mathrm{~K})$ we find $\sigma_{\mathrm{x}}=6.12 \times 10^{-30} \mathrm{~m}^{2}$. Doubling the mass $\left(P_{0} / g\right)$, results in $T=313.2 \mathrm{~K}$, or a climate sensitivity of the entire atmosphere of $\Delta T / \Delta M=(25 \mathrm{~K}) /\left(10^{4} \mathrm{~kg} / \mathrm{m}^{2}\right)$. Likewise, doubling $\sigma_{\mathrm{x}}$ which, remember, is the weighted average of the capture cross sections of all molecules in the atmosphere, for instance by doubling the fraction of the only agent with non-zero absorption (from $0.035 \%$ to $0.070 \%$ ), will increase the temperature by 25.1 degrees. This result is exactly equal to the one we found for a homogeneous atmosphere! Although the calculations are much more complicated, the results are the same. That means that for all purposes, we can consider the atmosphere-temperature, pressure and density curves-in any shape we want, the results will be the same, the exact shape does not matter. That will facilitate our analysis. We do not need supercomputers to calculate things, we can use a pocket calculator.

These results are phenomenologically equal to the case of the closed-box Beer-Lambert model. It has the same linear radiation forcing behavior of $D(0)$, depending linearly on total amount of absorbant in the atmosphere. For instance, doubling the total atmosphere with all constituents in it doubles $P_{0}$ and that doubles the downward radiation (Equation (45)). Moreover, the dependence of temperature on total amount of $\left[\mathrm{CO}_{2}\right]$ is likewise of the same behavior.

If $\mathrm{CO}_{2}$ is the only gas contributing to the greenhouse effect in the atmosphere, the above number would imply a climate sensitivity of $\Delta T / \Delta\left[\mathrm{CO}_{2}\right]=$ $(25.1 \mathrm{~K}) /(350 \mathrm{ppm})=72 \mathrm{mK} / \mathrm{ppm}$. Now, estimates are given that $\mathrm{CO}_{2}$ contributes to about $3.62 \%$ of the greenhouse effect [18]. Substituting $\sigma_{\mathrm{x}}^{\prime}=1.0362 \sigma_{\mathrm{x}}$ gives a temperature of $289.2 \mathrm{~K}$, and thus a climate sensitivity of $s=\Delta T / \Delta\left[\mathrm{CO}_{2}\right]=$ $(1.0 \mathrm{~K}) /(350 \mathrm{ppm})=2.9 \mathrm{mK} / \mathrm{ppm}$.

Figure 7 shows these radiation densities in the atmosphere. We can see that about $236 \mathrm{~W} / \mathrm{m}^{2}$ comes out from the top of the atmosphere to the universe and $154.83 \mathrm{~W} / \mathrm{m}^{2}$ comes out from the bottom of the atmosphere to the surface (solid 


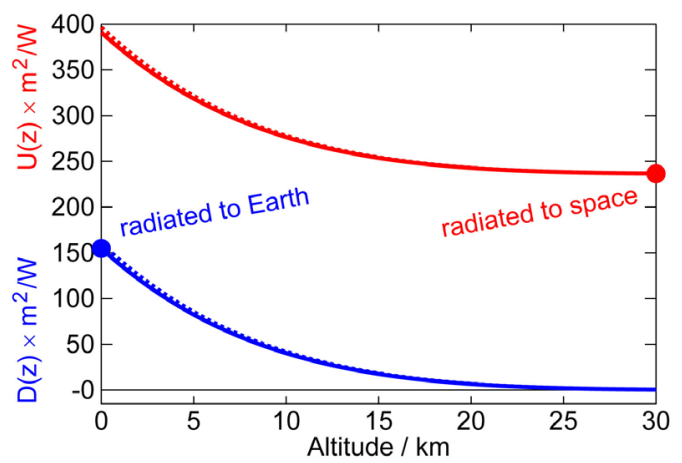

Figure 7. Radiation density in the atmosphere. The red curve represents the upward radiation $(U(z))$ and the blue curve represents the downward radiation $(D(z))$. Increasing $\left[\mathrm{CO}_{2}\right]$ by a factor $2\left(\sigma_{\mathrm{x}}\right.$ by $3.62 \%$ ) results in the dashed curves.

curve). These are effective black-body temperatures of $254 \mathrm{~K}$ and $229 \mathrm{~K}$, respectively. When we increase the capture cross section $\sigma_{\mathrm{x}}$ by $3.62 \%$, the downward radiation increases to $160.44 \mathrm{~W} / \mathrm{m}^{2}$. That implies a forcing of $5.605 \mathrm{~W} / \mathrm{m}^{2}$, or $w=16.0 \mathrm{~mW} / \mathrm{m}^{2}$ per $\mathrm{ppm}$.

In a separate section, the transient behavior is discussed. However, it must be pointed out that the idea of multiple-absorption-and-emission presented here very much resembles the solar atmosphere. A photon emitted at the heart of the Sun takes millions of years to reach the surface in a random-walk scenario (estimates differ several orders of magnitude). One might thus think that a photon in the terrestrial atmosphere, governed by the same process, will also take a long time. This however is not true. The time it takes is the number of steps to reach the edge of the atmosphere, multiplied by the time per step; with lifetime of states short, the time per step is governed by the speed of light. The number of steps in a random walk is quadratic with the linear distance. Yet, as we have seen, 28.5\% light traverses the atmosphere unhindered, making the total number of steps of the order unity, and the traveling speed close to the speed of light; no significant delay is expected because of this mechanism.

\subsection{Failure of the Model}

Now, as mentioned before, we have to conclude that this entire idea of an analysis is wrong, and is only performed here to show what values we might get on basis of the (faulty) analysis. Because of before-mentioned heat creep and thermodynamic equilibrium in general, it does not matter where and how the planet is heated (where radiation is absorbed, etc.), what matters is only the total amount of power, $(1-a) S$ absorbed in the Earth system and $U(h)$ emitted by it, in equilibrium they are equal. For ease of calculation we put it all at the surface, but it makes no difference whatsoever. Thus, as a consequence, and even more important to observe, radiation coming from the surface, $U(0)$, or anywhere else from the atmosphere itself should not be taken into account, since it does not add anything to the total energy input, it is merely a way of redistribution of internal heat, just like transport by convection and evaporation, etc., the 
final distribution which is given by the equations given here based on ideal gas laws. The idea that gases in the atmosphere work as some sort of "mirror" to reflect heat back to the surface is incorrect, because a very efficient and functional "mirror" already exists: Because the atmosphere is in thermodynamic equilibrium (as evidenced by the perfect fit of thermodynamic-equilibrium equations to empirical reality, see Figure 6) adding a heat flux $F$ to the system from the top of the atmosphere to the bottom (see Figure 8)-for instance an absorption of IR and reemission downwards-will be fully counteracted by an equal flux $F$ from the bottom of the atmosphere to the top. The net effect will always be zero! Adding internal radiation to the radiation balance only, and ignoring the annulling other effects, would be allowing an atmosphere to boot-strap itself, heating itself somehow. No object can heat itself to higher temperatures, when it is already in thermal equilibrium. The only radiation that matters is the one coming from the Sun, and it does not matter where and how it enters into the heat balance of the atmosphere. Heat creep (convection, evaporation and radiation) will redistribute the heat to result in the distribution given here (Figure 6); According to Sorokhtin $66.56 \%$ by convection, $24.90 \%$ by condensation and $8.54 \%$ by radiation [19]. An added process (by multiple absorption-emission) might, at best, speed up the equilibration. There is nothing $\mathrm{CO}_{2}$ would add to the current heat balance in the atmosphere, if the outward radiation no longer comes from the surface of the planet, but from a layer high up in the atmosphere. As long as the radiation does not come from the surface, making the layer blacker (more emissive) will radiate- "mirror"-more heat downwards, which is irrelevant (since it will only speed up the rate of thermalization), but also more heat upwards ( $F$ in Figure 8), cooling down that layer and thermodynamically the surface layer and the entire planet with it! Opening a radiative channel to the cold universe will rather cool an object.

On the other hand, the procedure imagined in the greenhouse framework might be as follows. Based on the optical properties $\sigma_{\mathrm{x}}$ of an average molecule of the atmosphere we determine the optical mass $M_{\bullet} \propto 1 / \sigma_{\mathrm{x}}$ of the layer needed at the top of the atmosphere to absorb (or emit) the amount of IR radiation, the layer optically visible in the IR from an observer outside the atmosphere. The so-called penetration depth (not in terms of distance, but mass).

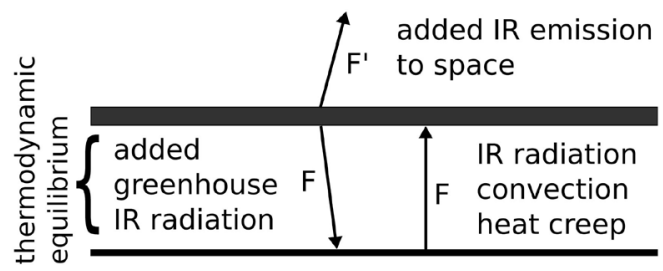

Figure 8. Adding an IR radiative emission $F$ from the radiative layer in the atmosphere down to the surface also adds a proportional radiation $F$ into space. The radiation downwards is compensated by an exact equal heat transfer $F$ upwards (comprised of things like convection, radiation and heat creep), to maintain thermodynamic equilibrium. The total effect is a cooling of the layer, a cooling which then percolates down to the surface. 
Given this mass $M_{.}$, we can determine the pressure at the bottom of this layer, $P_{\bullet}=g M_{\bullet}$. We know also the temperature $T_{\bullet}$ at the bottom of this layer (namely $254 \mathrm{~K}$ ), where the outward radiation effectively comes from, as discussed before. And we then know enough to calculate all the curves of the entire atmosphere above ( $M$. mass) as well as below this layer ( $M-M$. mass). As an example, the layer of the optical IR source in Figure 6 is at an altitude $z$. of ca. $6 \mathrm{~km}$, where the pressure is $500 \mathrm{hPa}$ and the mass of the layer above it ca. $M_{.}=P_{\bullet} / g=5000 \mathrm{~kg}$. Doubling the absorption $\sigma_{\mathrm{x}}$ of the average molecule (for instance by doubling the concentration of the only optically-active constituent) will halve the necessary mass $M_{\text {. }}$. It will thus only produce $250 \mathrm{hPa}$ of pressure at a certain new level $z_{\text {. }}$. The two only unknown variables, height $z$. and temperature at the ground $T_{0}$, can be found by solving the Equations (38) and (36), respectively

$$
\begin{gathered}
P_{\bullet}=g M_{\bullet}=P_{0}\left(1-\frac{g}{c_{p} T_{0}} z \cdot\right)^{m c_{\mathrm{p}} / k}=250 \mathrm{hPa}, \\
T_{\bullet}=T_{0}-\frac{g}{c_{p}} z \cdot=254 \mathrm{~K} .
\end{gathered}
$$

Solving the general case, we find

$$
T_{0}=T_{\bullet}\left(\frac{P_{0}}{g M_{\bullet}}\right)^{k / m c_{\mathrm{p}}},
$$

with $T_{\bullet}=254 \mathrm{~K}, \quad P_{0}=g M=1013.2 \mathrm{hPa}, \quad c_{\mathrm{p}}=1.51 \mathrm{~kJ} / \mathrm{kg} \cdot \mathrm{K}, \quad m=4.82 \times 10^{-26}$

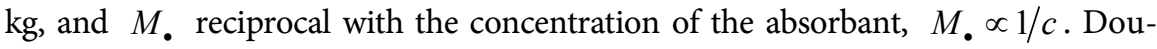
bling the specific absorption coefficient-doubling the average absorption cross section $\sigma_{\mathrm{x}}$-will halve $M$. and this will increase the surface temperature $T_{0}$ by $40.5^{\circ} \mathrm{C}$.

The problem with this analysis is that we again go back to the assumption that the distribution of heat in the atmosphere is the result of radiation balances, while, as argued above, the atmosphere is not governed by radiation, but by thermodynamics. The same reasoning thus applies to the layers above the "radiating layer", where the temperature is $T_{\bullet}$, as it does for the layers below. A radiation analysis is provenly wrong and we should not fall back to this way of thinking. The atmosphere must be analyzed by thermodynamic laws in its entirety.

Contrastingly often the atmosphere is analyzed by radiation laws in its entirety. This, however, is also not possible. In a radiation analysis, absorption by a layer at $z$ is not depending on temperature (we assume it manages to absorb everything), and only depends on the density of a layer: $\propto \rho(z) \sigma_{\mathrm{x}}$. The emission, however, is depending on the density of emitters and the temperature, for instance the Stefan Boltzmann function $\left(f(T)=\sigma T^{4}\right): \propto \rho(z) f(T(z))$. In dynamic equilibrium, absorption is equal to emission, and thus the density $\rho(z)$ factors out and cannot be determined on basis of radiation balance. We can only simulate the atmosphere as is, without knowing where it came from. 
Meaning, given the atmosphere, we would be able to calculate the radiation balance therein, for instance the radiation curves in a homogeneous atmosphere, $\rho(z)$ constant (Figure 4(b)), or thermodynamic atmosphere (Figure 7). It is highly unsatisfying when we must assume things.

We are, for instance, not able to reconstruct the empirical temperature profile of the atmosphere. The radiation balance analysis, by ignoring the fact that the atmosphere in a one-sided-open box system, is incapable of supplying a phenomenological and analytical description of the system. The thermodynamic approach, on the other hand, has difficulty explaining why the surface has heated up at all; the argumentation above is that blackening the atmosphere might make it cool down. We are obviously in need of a better descriptive model. This will be presented now.

\section{Thermodynamic-Radiative Atmospheric Model}

This brings us to the final model that will be presented here. It is based on combining the thermodynamic and radiative analyses given above.

Considering the fact that the atmosphere is in thermodynamic equilibrium, we must assume that absorbed radiation does get assimilated by the heat bath, just like in classic Beer-Lambert theory. Radiation absorbed is distributed instantaneously all over the atmosphere and the surface. The surface emits with $\sigma T_{0}^{4}$, a part $\lambda$ is passing directly to the universe unhindered, $1-\lambda$ is absorbed. The atmosphere emits too; the total emissivity equal to $\varepsilon$.

Since, for radiation properties, it does not matter where the absorbing/emitting mass resides $(z)$, but only how much radiation it receives and what temperature it has, we start by describing the atmosphere $\rho$ and $T$ not as a function of height $z$, but as a function of temperature $T$. The thermodynamic equations (36) and (39) combine into

$$
\rho^{\prime}(T)=\frac{c_{\mathrm{p}}}{g} \times \frac{m P_{0}}{k T_{0}}\left(\frac{T}{T_{0}}\right)^{m c_{\mathrm{p}} / k-1}\left(\text { unit }: \mathrm{kg} / \mathrm{K} \cdot \mathrm{m}^{2}\right) .
$$

The first factor comes from the unit conversion $\left(\mathrm{kg} / \mathrm{m}^{3}\right.$ to $\left.\mathrm{kg} / \mathrm{Km}{ }^{2}\right)$. The "density" (for lack of a better word) is not mass per volume, but mass per area per temperature slice. The integral for the entire atmosphere is again $\int_{0}^{T_{0}} \rho^{\prime}(T) \mathrm{d} T=$ $P_{0} / g=M$. We use here a different symbol $\rho^{\prime}$ to remind us that the units are different.

The total energy in the atmosphere can easily be calculated. Since in thermodynamic equilibrium all air packages have the same specific energy given by $c_{\mathrm{p}} T+g z$, independent of $z$, all mass must have specific energy (per $\mathrm{kg}$ ) equal to that at $z=0$, namely $c_{\mathrm{p}} T_{0}$. The total thermodynamic energy of the atmosphere is then $E_{\text {total }}=c_{\mathrm{p}} T_{0} M$. The atmosphere tries to shed energy by radiation, and receives energy from the surface. The surface also tries to shed energy, either by radiation into the universe, or by transfer to the atmosphere somehow (conduction, etc.). This is a intricate interplay of energy transfer.

We go back to the Beer-Lambert analysis. Once again, this is justified by the 
assumption that internal absorption is simply recycled back into the system. The heat is rapidly distributed to maintain thermodynamic equilibrium.

Radiation leaving the surface $\sigma T_{0}^{4}$ is partly absorbed by the atmosphere and partly transmitted. The absorbed energy goes back to the heat bath $c_{\mathrm{p}} T_{0} M$ and is redistributed therein. It does not heat up this bath (that would be counting the solar radiation twice), but reabsorbed energy is heat that was not allowed to escape the system and is thus blocked from cooling it.

The absorbed part of the radiation is proportional to the concentration and absorption coefficient,

$$
\frac{\mathrm{d} U(T)}{\mathrm{d} T}=-\sigma_{x} \frac{\rho^{\prime}(T)}{m} U(T) .
$$

As we have seen, the transmittance of such a system is given by (Equation (5))

$$
\begin{aligned}
U(h) & =U(0) \exp \left(-\frac{\sigma_{\mathrm{x}}}{m} \int_{0}^{T_{0}} \rho^{\prime}\left(T^{\prime}\right) \mathrm{d} T^{\prime}\right) \\
& =\sigma T_{0}^{4} \exp \left(-\frac{\sigma_{\mathrm{x}} M}{m}\right) .
\end{aligned}
$$

Now, radiation emitted by the atmosphere itself follows a similar path. Each layer emits with an emissivity $\varepsilon=\sigma_{\mathrm{x}}$, at a temperature $T$ by a concentration $\rho^{\prime}(T) / m$. What goes up is:

$$
\begin{aligned}
v(T) & =\frac{1}{2} \frac{\rho(T)}{m} \sigma_{\mathrm{x}} \sigma T^{4}\left(\text { unit }: \mathrm{W} / \mathrm{K} \cdot \mathrm{m}^{2}\right) \\
& =\frac{c_{p} P_{0} T_{0}^{3} \sigma_{\mathrm{x}} \sigma}{2 g k}\left(\frac{T}{T_{0}}\right)^{m c_{\mathrm{p}} / k+3} .
\end{aligned}
$$

This passes through layers with temperatures $T$ to 0 , and densities $\rho^{\prime}(T)$ through 0 . What leaves the atmosphere is

$$
\begin{aligned}
v(h, T) & =v(T) \exp \left(-\frac{\sigma_{\mathrm{x}}}{m} \int_{0}^{T} \rho\left(T^{\prime}\right) \mathrm{d} T^{\prime}\right) \\
& =\frac{c_{\mathrm{p}} P_{0} T_{0}^{3} \sigma_{\mathrm{x}} \sigma}{2 g k}\left(\frac{T}{T_{0}}\right)^{m c_{\mathrm{p}} / k+3} \exp \left(-\frac{\sigma_{\mathrm{x}} M}{m}\left[\frac{T}{T_{0}}\right]^{m c_{\mathrm{p}} / k}\right) .
\end{aligned}
$$

Integrating over all layers [20]:

$$
\begin{aligned}
V(h) & =\int_{0}^{T_{0}} v\left(h, T^{\prime}\right) \mathrm{d} T^{\prime} \\
& =\frac{\sigma T_{0}^{4}}{2}\left(\frac{\sigma_{\mathrm{x}} M}{m}\right)^{-4 k / m c_{\mathrm{p}}} \times \gamma\left(1+\frac{4 k}{m c_{\mathrm{p}}}, \frac{\sigma_{\mathrm{x}} M}{m}\right) .
\end{aligned}
$$

(With the two-argument $\gamma$ the incomplete-gamma function [21]). This is the energy emitted by the atmosphere that comes out from the top. We see that if not all radiation comes out, the energy of the system $\left(E_{\text {total }}=c_{\mathrm{p}} T_{0} M\right)$ increases-the surface temperature $T(z=0)=T_{0}$ increases-until the outward radiation $U(h)+V(h)$ equals the inward radiation $(1-a) S$.

We can now make a plot of the total radiation coming out of the atmosphere as a function of the total optical depth the atmosphere $\left(\tau=\sigma_{\mathrm{x}} M / m\right)$. Combining Equations (52) and (55), Figure 9 shows the fraction 


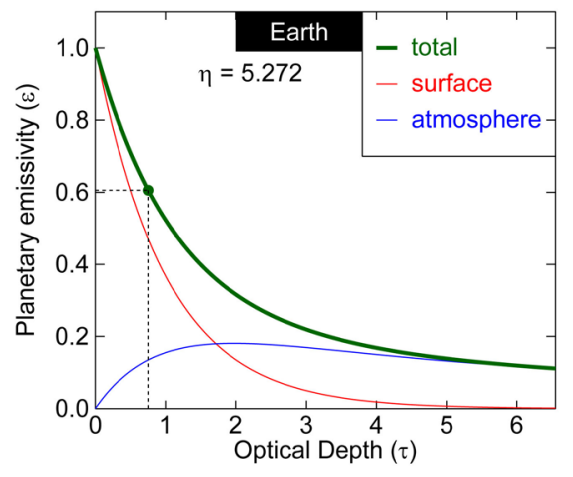

(a)

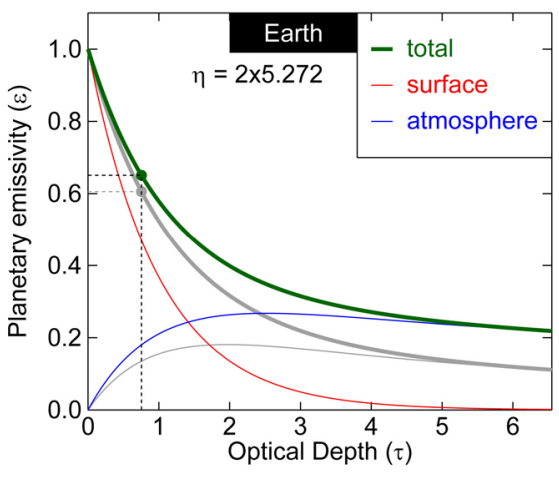

(b)

Figure 9. Left: Earth planetary emissivity $\epsilon$ of the fraction of the radiation emitted by the surface that is escaping from the top of the atmosphere. 1 is like a black body, 0 is white. The red curve is the contribution from the surface and the blue curve from the atmosphere. The sum is $\epsilon$, shown by the green curve. Right: The effect of augmenting the specific heat $c_{\mathrm{p}}$ of air is an increment of the planetary emissivity and thus cooling of the surface if $\eta=m c_{\mathrm{p}} / k$ increases, but also a heating up of the atmosphere from where now more radiation originates. (Gray lines are the situation of left figure, colored lines are for a doubling of $c_{\mathrm{p}}$ ).

$$
\begin{aligned}
\epsilon & \equiv \frac{U(h)+V(h)}{U(0)} \\
& =\exp \left(-\frac{\sigma_{\mathrm{x}} M}{m}\right)+\frac{1}{2}\left(\frac{\sigma_{\mathrm{x}} M}{m}\right)^{-4 k / m c_{\mathrm{p}}} \times \gamma\left(1+\frac{4 k}{m c_{\mathrm{p}}}, \frac{\sigma_{\mathrm{x}} M}{m}\right) \\
& =\exp (-\tau)+\frac{1}{2}(\tau)^{-4 / \eta} \times \gamma(1+4 / \eta, \tau) .
\end{aligned}
$$

which is the planetary emissivity $\epsilon$, as a function of optical optical depth $\tau=$ $\sigma_{\mathrm{x}} M / m$, and thermodynamic molecular heat capacity $\eta=m c_{\mathrm{p}} / k$. Note, the planetary emissivity can also be described as the ratio of the total radiation emitted by the planet (equal to the black body temperature emission) and the radiation emitted by the surface:

$$
\epsilon=\frac{\sigma T_{\mathrm{bb}}^{4}}{\sigma T_{0}^{4}}=\left(\frac{T_{\mathrm{bb}}}{T_{0}}\right)^{4},
$$

or, in other words

$$
T_{0}=\frac{T_{\mathrm{bb}}}{\sqrt[4]{\epsilon}}
$$

with $\epsilon$ given by Equation (56).

The effect of the atmosphere can be found by knowing the thermodynamic parameter and the optical parameter, more precisely the molecular heat capacity, $\eta=m c_{\mathrm{p}} / k$, and optical depth, $\tau=\sigma_{\mathrm{x}} M / m$. The latter is complicated, since it is not simply a matter of a linear function of $M$. We have to know the complete spectrum. But before we continue, it has to be pointed out that the emissivity Equation (Equation (56)) is a monotonously decreasing function of $\tau$ for any 
value of $\eta$. That means that increasing the optical depth of the atmosphere will always reduce the emissivity and increase the surface temperature, in contrast to earlier thoughts we had.

Reabsorbed radiation goes back to the heat bath and gets a second change to be radiated out to the universe, maybe through another channel: Emitted at a wavelength for which the atmosphere is more transparent, or maybe from a place higher up in the atmosphere.

We can show here what happens in a simplified picture. If we assume the thermodynamic properties $\eta$ of the atmosphere constant, and also assume for the moment changes of $\tau$ in the incomplete gamma function to be irrelevant, we can schematically describe the effect of changes in absorption. Figure 10 shows a schematic representation of some imaginary cases. It shows the absorptivity (that ranges from $0=$ "fully transparent" to $1=$ "fully opaque") for wavelengths in the emission spectrum (represented by a semicircle). Case (1) shows a fully transparent atmosphere. Obviously, doubling the atmosphere has no effect on the temperature, which stays at $T_{0}=T_{\mathrm{bb}}$. Case (2) is an atmosphere equally opaque at all relevant wavelengths. All channels are nearly closed (they can never be fully closed; Beer-Lambert is exponential). Increasing one of the responsible absorbing agents has a large effect, because the transparency is strongly dependent on $\tau$ that is now linear with $M$. Likewise case (3), which is similar to case (4), but absorbs only half. Changes in concentration of atmospheric agent

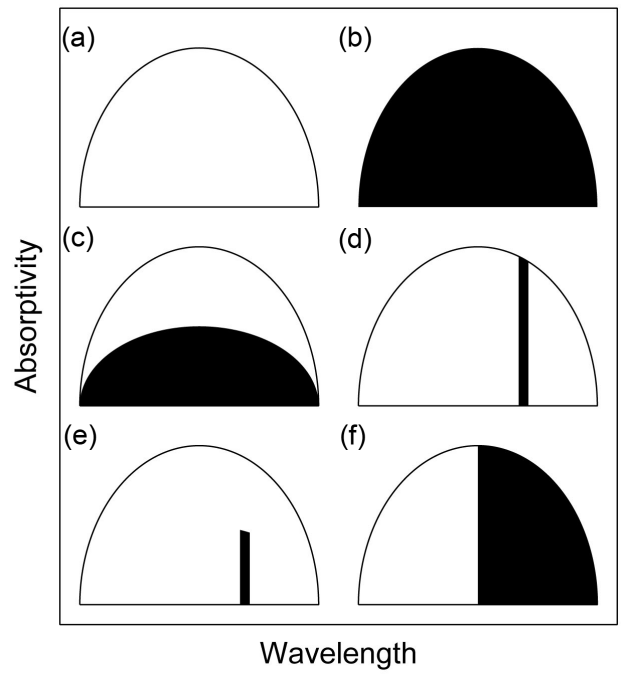

Figure 10. Schematic representation of some cases of greenhouse effect. The semicircle represents the emission spectrum as a function of wavelength. The absorptivity is 0 when fully transparent and 1 when fully opaque. The effect of increasing absorbant in the atmoshpere are: (a) Fully transparent atmosphere, $T_{0}=T_{\mathrm{bb}}$. Increasing constituents of the atmosphere has no effect; (b) Fully opaque, increases in the atmosphere have strong effects; (c) Even though not saturated, the effects of changes are stills severe; (d) A fully saturated spectrum that covers only part of the emission. Changes have no effect; (e) Not fully saturated, changes still have some effect, but are not so big; (f) The abosrbant covers half the spectrum, but is saturated. Increasing the concentration of the absorbant has no effect. 
have a large effect. In case (5) the absorption spectrum covers only a small part of the emission spectrum (for instance 1\%) and that part is nearly saturated. Doubling the responsible agent will have nearly no effect, the transmissivity of the atmosphere stays constant (for instance 99\%). In case (6) the absorption line is not yet saturated and increases still contribute to the greenhouse effect, though not much. Transmission can go down from say $99.5 \%$ to $99.0 \%$. In case (7) the absorption covers half of the emission spectrum. Increasing the concentration of the absorbing agent will have no effect and the emissivity stays at 0.5 .

\section{Comparing to Reality}

Unfortunately, we have no planet B and we cannot do scientific experiments with Earth. However, we can still see how well the theory fares. Fortunately, nature throws interesting data in our direction, which we can study. These can be found on Earth, but also on other planets (there are "Planet Bs" in our solar system). Beginning with Mars.

\subsection{Mars}

The relevant parameters of the NASA Mars Fact Sheet [22] are given in Table 4. The mass per area can be found by $M_{\hat{\delta}}=P_{0, \hat{\delta}} / g_{\hat{\delta}}=171.42 \mathrm{~kg} / \mathrm{m}^{2}$. Because most of the atmosphere consists of $\mathrm{CO}_{2}$ (sic), the average molecular mass of molecules is close to the value of $\mathrm{CO}_{2}(44.01 \mathrm{~g} / \mathrm{mol})$, the fact sheet gives $43.34 \mathrm{~g} / \mathrm{mol}$ [23]. Therefore, the atmosphere has $3.955 \mathrm{kmol} / \mathrm{m}^{2}$ of molecules. $95.32 \%$ of that is $\mathrm{CO}_{2}$, that is $3.770 \mathrm{kmol} / \mathrm{m}^{2}$. That is much more than above any point on Earth. Yet, the effect of all that $\mathrm{CO}_{2}$ is unmeasurable. The black body (atmosphereless) temperature is $T_{\mathrm{bb}, \hat{\delta}}=209.8 \mathrm{~K}$, which can be calculated on basis of the solar irradiance $W_{\delta}=586.2 \mathrm{~W} / \mathrm{m}^{2}$ and the albedo of Mars $\left(a_{\delta}=0.250\right)$ : $\sigma T_{\mathrm{bb}, \hat{\delta}}^{4}=\left(1-a_{\delta}\right) W_{\delta} / 4$. The real temperature of the Mars surface is just this 210 $\mathrm{K}$, making the measured greenhouse effect of orders-of-magnitude-more- $\mathrm{CO}_{2}$ than-on-Earth zero within the measurement error. Note also that the emission spectrum of a black body at $210 \mathrm{~K}$ has a maximum very close to the $\mathrm{CO}_{2}$ spectral line shown in Figure 11.
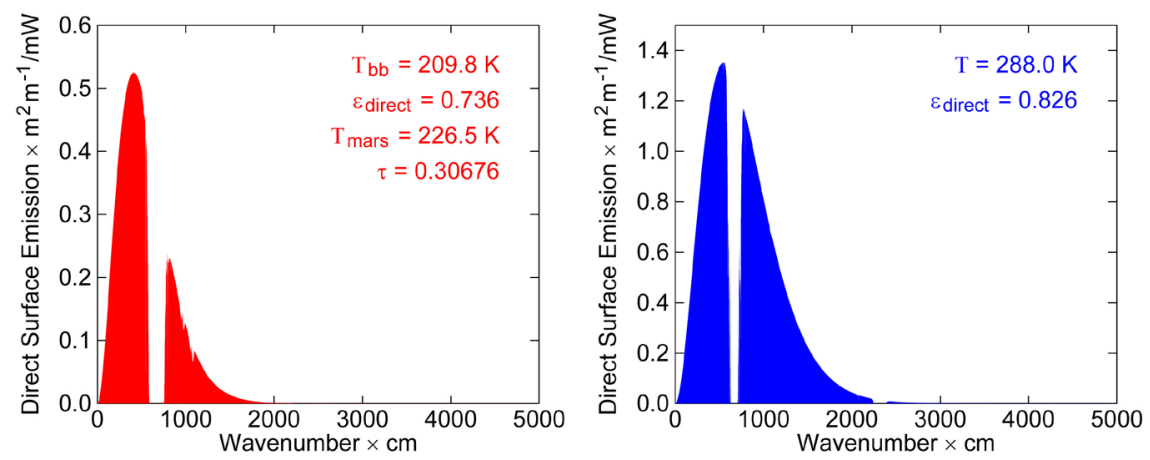

Figure 11. Mars (left) and Earth (right) planetary direct-emission spectrum based on the HITRAN spectrum of $\mathrm{CO}_{2}$ of Earth [25] with the absorption coefficient for Mars multiplied by 30 . 
Table 4. NASA Fact Sheet for Mars [22] and Venus [24], except $c_{\mathrm{p}}$.

\begin{tabular}{|c|c|c|}
\hline Parameter & Mars & Venus \\
\hline Black body temperature & $T_{\mathrm{bb}, \delta}=209.8 \mathrm{~K}$ & $T_{\mathrm{bb}, q}=226.6 \mathrm{~K}$ \\
\hline Average temperature & $T_{0, \delta}=210 \mathrm{~K}$ & $T_{0, q}=737 \mathrm{~K}$ \\
\hline Bond albedo & $a_{\text {子 }}=0.250$ & $a_{q}=0.77$ \\
\hline Surface gravity & $g_{\delta}=3.71 \mathrm{~m} / \mathrm{s}^{2}$ & $g_{q}=8.87 \mathrm{~m} / \mathrm{s}^{2}$ \\
\hline Solar irradiance & $W_{\delta}=586.2 \mathrm{~W} / \mathrm{m}^{2}$ & $W_{q}=2601.3 \mathrm{~W} / \mathrm{m}^{2}$ \\
\hline Atmospheric $\mathrm{CO}_{2}$ & {$\left[\mathrm{CO}_{2}\right]_{\delta}=95.32 \%$} & {$\left[\mathrm{CO}_{2}\right]_{\varphi}=96.5 \%$} \\
\hline Surface pressure & $P_{0, \delta}=6.36 \mathrm{hPa}$ & $P_{0, q}=9.2 \mathrm{MPa}$ \\
\hline \multirow[t]{2}{*}{ Mean molecular weight } & $m_{\delta}=43.34 \mathrm{~g} / \mathrm{mol}$ & $m_{q}=43.45 \mathrm{~g} / \mathrm{mol}$ \\
\hline & $m_{\delta}=7.197 \times 10^{-26} \mathrm{~kg}$ & $m_{q}=7.215 \times 10^{-26} \mathrm{~kg}$ \\
\hline Specific heat & $c_{\mathrm{p}, \hat{\mathrm{s}}}=0.844 \mathrm{~kJ} / \mathrm{kg} \cdot \mathrm{K}^{*}$ & $c_{\mathrm{p}, q}=0.844 \mathrm{~kJ} / \mathrm{kg} \cdot \mathrm{K}^{*}$ \\
\hline
\end{tabular}

$*$ value of $\mathrm{CO}_{2}$.

Of course, what is important is not so much how much $\mathrm{CO}_{2}$ is absorbing, but how much is the atmosphere in its entirety absorbing. Better to say, how much it is letting through. Even with $\mathrm{CO}_{2}$ fully saturated, nearly all radiated heat easily escapes the atmosphere. A tiny unmeasurable effect remains. Now, doubling it will have no effect. Imagine $1 \%$ of the spectrum is covered, in which part $90 \%$ of the radiation is absorbed. Thus $99.1 \%$ of all radiation escapes. Doubling this constituent will make the absorption in that 1\% part only go to $99 \%$; still $99.01 \%$ of all radiation escapes. In this particular case of Mars, $\mathrm{CO}_{2}$ has little effect in whatever quantity it is in the atmosphere. This resembles situation (d) in Figure 10. We can calculate how much it is.

Although the Martian greenhouse effect is zero within the margin of error, let's assume here a value equal to $0.2 \mathrm{~K}$. The emissivity of Mars is then $\epsilon=$ $[(209.8 \mathrm{~K}) /(210.0 \mathrm{~K})]^{4}=0.99620$. Using the specific heat value of $\mathrm{CO}_{2}$ (Table 3), $c_{\mathrm{p}}=0.844 \mathrm{~kJ} / \mathrm{kg} \cdot \mathrm{K}$, a molecular mass of $m=7.20 \times 10^{-26} \mathrm{~kg}$ the thermodynamic parameter $\eta=m c_{\mathrm{p}} / k=4.401$.

However, using high-quality optical spectroscopy data such as HITRAN [25] assuming only $\mathrm{CO}_{2}$, and multiplying its optical depth of just $\mathrm{CO}_{2}$ by a factor 30 , convoluting the resulting spectrum with a $210 \mathrm{~K}$ black-body emission, we find a direct emission ("filling factor" of the emission spectrum) of $73.6 \%$, see Figure 11 (absorption of 26.4\%), which is represented by the red dot on the red curve in Figure 12. From the atmosphere comes another $4.8 \%$ radiation, giving a total emissivity of $\epsilon=0.784$. This would imply a surface temperature of $(209.8 \mathrm{~K}) / \sqrt[4]{0.784}=223.0 \mathrm{~K}$. It is obvious that the theory fails miserably already at its first simple test. 

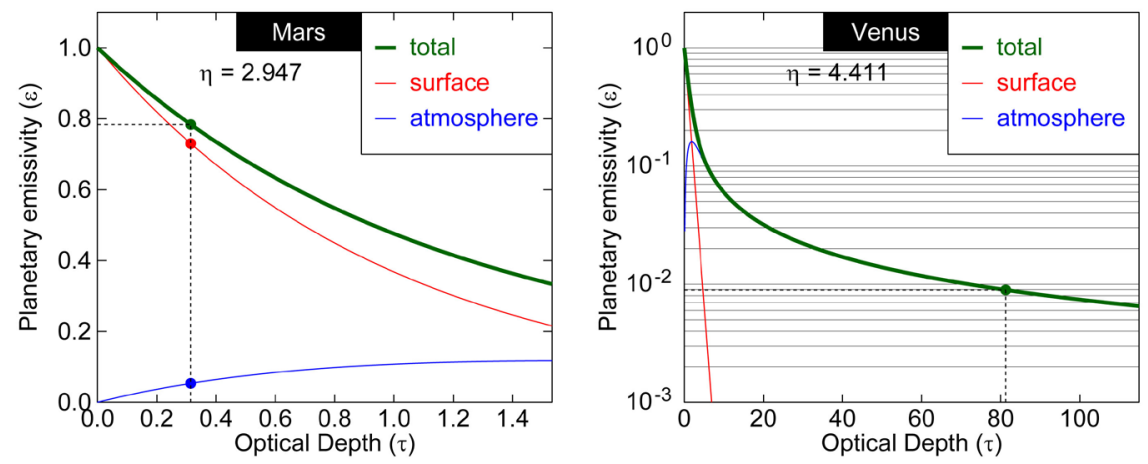

Figure 12. Mars (left) and Venus (right, log scale) planetary emissivity $\epsilon$ of the fraction of the radiation emitted by the surface that is escaping from the top of the atmosphere. 1 is like a black body, 0 is white. The red curve is the contribution from the surface and the blue curve from the atmosphere. The sum is $\epsilon$, shown by the green curve.

\subsection{Earth}

Ångström, in his classical work, wrote "[...] it is clear, first, that no more than about 16 percent of earth's radiation can be absorbed by atmospheric carbon dioxide, and secondly, that the total absorption is very little dependent on the changes in the atmospheric carbon dioxide content, as long as it is not smaller than 0.2 of the existing value" [5]. This basically states that there is no further contribution to the greenhouse effect from $\mathrm{CO}_{2}$ for concentrations above, about, $60 \mathrm{ppm}$. It is another way of saying that a radiation window that is closed cannot further contribute to greenhouse effect. That is, as long as there are other windows still open. This is not true, however, because absorption lines have tails that can never saturate, absorption can be much larger. A HITRAN simulation of only $\mathrm{CO}_{2}$ absorption (with concentrations as found in the Earth atmosphere) results in absorption of $17.4 \%$ (See Figure 11; direct emission 82.6\%), close to the value estimated by Ångström. Yet, as we have seen, for Mars, with 30 times more $\mathrm{CO}_{2}$, this absorption is $26.4 \%$. It shows the complexity of the subject. This manuscript is not about numerical simulations, but about analytical understanding of the greenhouse effect. We will now make an empirical estimation here in the framework of our analytical model.

The real emissivity of Earth (surface plus atmosphere) can easily be determined on basis of empirical data, according to Equation (57), where we defined the emissivity through the ratio of radiation coming out to the radiation emitted by the surface:

$$
\epsilon=\left(\frac{254 \mathrm{~K}}{288 \mathrm{~K}}\right)^{4}=0.605
$$

Taking the thermodynamic parameters $\left(m, c_{\mathrm{p}}\right)$ as constant and as used before (Table 3), from the equations we find that this value occurs for an optical depth of $\tau=0.754$ (See the green dot of Figure 9). We can even see that $77.7 \%(0.470)$ of the radiation going into space comes from the surface and $22.3 \%(0.135)$ from the atmosphere. This contrasts the notion mentioned earlier that the radiation 
comes from $6 \mathrm{~km}$ altitude (most still comes from the surface), and also contrasts the values of $28.5 \%$ direct emission found earlier for a radiation-only atmosphere. Yet, we also see that if the opacity of the atmosphere increases, more radiation, also in absolute terms, comes from the atmosphere. This is the cooling effect of the atmosphere described earlier.

We would have to know the spectrum of the atmosphere, but we can make a worst-case scenario. That is, assuming the spectrum is flat (situation (c) in Figure 10). The optical depth is then proportional to the density. Doubling the optical depth (for instance by doubling the absorption coefficient $\sigma_{\mathrm{x}}$ ) brings $\epsilon$ down to 0.398 , and the surface temperature, to compensate for this increased whiteness of the planet, raises the temperature to $T_{0}^{\prime}=320.0 \mathrm{~K}$. If all greenhouse effect is caused by $\mathrm{CO}_{2}$ it implies a climate sensitivity of $s \equiv \Delta T / \Delta\left[\mathrm{CO}_{2}\right]=$ $(32.0 \mathrm{~K}) /(350 \mathrm{ppm})=91 \mathrm{mK} / \mathrm{ppm}$. Now, estimates are given that $\mathrm{CO}_{2}$ contributes to about $3.62 \%$ of the greenhouse effect [18]. We thus get a final estimated open-loop worst case climate sensitivity of

$$
s=\frac{\mathrm{d} T}{\mathrm{~d}\left[\mathrm{CO}_{2}\right]}=3.3 \mathrm{mK} / \mathrm{ppm} .
$$

The radiative forcing can then be estimated. The full effect of $350 \mathrm{ppm}$ would be $\epsilon$ lowering from 0.605 to 0.398 . But we attribute only $3.62 \%$ to $\mathrm{CO}_{2}$, that is $7.666 \times 10^{-3}$ to $350 \mathrm{ppm}$, that is $\mathrm{d} \epsilon / \mathrm{d}\left[\mathrm{CO}_{2}\right]=2.2 \times 10^{-5} / \mathrm{ppm}$. Out comes less $2.2 \times 10^{-5} \sigma(288 \mathrm{~K})^{4}$ that must go to the surface (base temperature $T_{0}$ ). So, the worst case radiative forcing of $\mathrm{CO}_{2}$ is

$$
w=8.6 \mathrm{~mW} / \mathrm{m}^{2} \text { per } \mathrm{ppm} .
$$

However, the situation is much closer to situation ( $\mathrm{f}$ ) of Figure 10. That is, a part of the spectrum emission is fully open, and the part of $\mathrm{CO}_{2}$ is as good as closed (shown black in the figure). This situation is depicted in Figure 13, with an open channel A and a closed channel B. Now, further closing the channel (B) that is already as good as closed has little-to-no effect, as long as a significant part of the rest of the spectrum is open. Note that this is not envisaged in a radiation balance analysis. In that model, once a heat package has "decided" to opt for a certain wavelength, the only way to make it out of the atmosphere is by multiple-emission-absorptions events, or crawling its way back to the surface. As such, adding $\mathrm{CO}_{2}$ to a closed channel still has a lot of impact. In the current thermodynamic-radiative model, absorbed radiation is given back to the heat bath that is the surface plus the atmosphere $\left(c_{\mathrm{p}} T_{0} M\right)$, from where it can have a second chance of escaping into the universe, by the same or by a different channel.

To say it in another way, the best-case climate sensitivity of $\mathrm{CO}_{2}$ is zero. The optical length of $\mathrm{CO}_{2}$ in the atmosphere is about $25 \mathrm{~m}$. That is, 25 meters up in the air the radiation emitted by the surface in the spectrum of $\mathrm{CO}_{2}$ is already attenuated by a factor $e$. In this $25-\mathrm{m}$ layer resides only $1 / 1773$ th part of the atmosphere (and $\mathrm{CO}_{2}$ ). The total transmission of the entire atmosphere is thus 


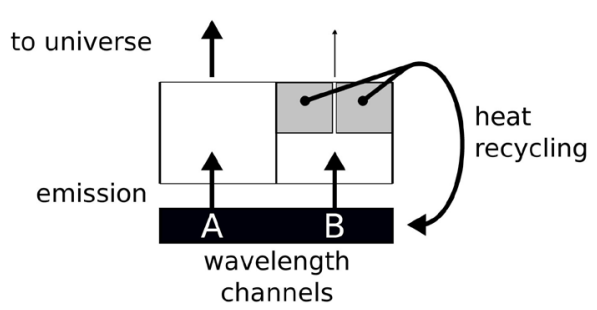

Figure 13. Schematic picture of what happens at Earth. Two channels, one (A) if fully open and one (B) is nearly closed. Closing B further has little-to-no effect.

$\exp (-1773)$ which any calculator shows as zero. Doubling the $\mathrm{CO}_{2}$ in the atmosphere, will have no measurable effect, $\exp (-3546) \approx 0$. Heat had no chance escaping to the universe through this channel, and now even less so. As long as there is a sliver of the emission spectrum for which the atmosphere is transparent the effect of doubling agents such as $\mathrm{CO}_{2}$ that have a spectrum that is close to saturation is close to nil. This is the lower limit of the effect. It is exactly the reason that gave rise to Global Warming in a radiative greenhouse model, namely the strong absorptivity of $\mathrm{CO}_{2}$, that undermines the existence of further greenhouse effects in a thermodynamic-radiative analysis.

Note that these are back-of-the envelope calculations done here. We are going to do simulations based on the HITRAN spectra [25] [26], but this study is fully analytical, without numeric tools. However, as we can see here, the thermodynamic-radiation theory can phenomenologically explain the temperature on Earth. Interesting also to note is that it does explain phenomenologically the terrestrial radiation spectra when considered for their latitude. Close to the equator, the radiation comes principally from the surface and the $\mathrm{CO}_{2}$ creates a hole in the emission spectrum there. However, heat is transported through the air to the poles that have a cold surface, but a relatively warm atmosphere; $\mathrm{CO}_{2}$ creates above Antarctica a peak in the emission spectrum [27]. At these wavelengths radiation comes from the atmosphere, that is above the equator, as well as above the poles, at roughly $220 \mathrm{~K}$.

\subsection{Sevilla-Córdoba-Granada}

In this thermodynamic analysis, the temperature at a point on the planet is for a certain radiative input $(1-a) S$, mainly determined by the altitude $z$. The radiative greenhouse effect states it mainly depends on the total amount of carbon dioxide floating above the point. To test these hypotheses, we can look at cities with the same or similar radiative solar input, at the same latitude on the planet, but at different altitudes. Without doing an exhaustive study, we take as example three neighboring cities in the south of Spain, namely Sevilla, Córdoba and Granada, each at a different elevation (Table 5).

Sevilla at an elevation of $34 \mathrm{~m}$ has an air mass of $P(34 \mathrm{~m}) / \mathrm{g}=10287 \mathrm{~kg} / \mathrm{m}^{2}$ above it, which is equal to $\left(10287 \mathrm{~kg} / \mathrm{m}^{2}\right) / m N_{A}=354 \mathrm{kmol} / \mathrm{m}^{2}$. Before the industrial era, at $\left[\mathrm{CO}_{2}\right]=280 \mathrm{ppm}(0.028 \%)$, this then translates to $99 \mathrm{~mol} / \mathrm{m}^{2}$ of $\mathrm{CO}_{2}$. Granada at 567 meters elevation has an air mass above it equal to 
Table 5. Comparative cities in the south of Spain. Data source: NOAA [28].

\begin{tabular}{cccc}
\hline Weather station & Coordinates & Elevation & Temperature in 2018 \\
\hline $\begin{array}{c}\text { SEVILLA SAN PABLO } \\
\text { (SPE00120512) }\end{array}$ & $37.4167 \mathrm{~N}, 5.8792 \mathrm{~W}$ & $34 \mathrm{~m}$ & $18.5^{\circ} \mathrm{C}$ \\
$\begin{array}{c}\text { CORDOBA AEROPUERTO } \\
\text { (SP000008410) }\end{array}$ & $37.844 \mathrm{~N}, 4.8458 \mathrm{~W}$ & $90 \mathrm{~m}$ & $18.8^{\circ} \mathrm{C}$ \\
$\begin{array}{c}\text { GRANADA AEROPUERTO } \\
\text { (SPE00120089) }\end{array}$ & $37.1894 \mathrm{~N}, 3.7892 \mathrm{~W}$ & $567 \mathrm{~m}$ & $15.9^{\circ} \mathrm{C}$ \\
\hline
\end{tabular}

$P(567 \mathrm{~m}) / g m N_{A}=332 \mathrm{kmol} / \mathrm{m}^{2}$ of which $0.042 \%, 140 \mathrm{~mol} / \mathrm{m}^{2}$, in 2019 is $\mathrm{CO}_{2}$. The question now is, why is Granada not much warmer in 2019 than Sevilla was in 1951? It is actually still colder. This seriously undermines the idea that carbon dioxide is determining the temperature on our planet. Figure 14 plots the temperature of these cities versus the carbon content above them. The linear regression quality parameter is $\mathrm{R}^{2}=0.21$. Meaning, temperature is not well correlated with $\left[\mathrm{CO}_{2}\right]$.

\subsection{Venus}

Similar to Mars is Venus. The relevant parameters from NASA's Venus Fact Sheet are given in Table 4. The mass density of the Venusian atmosphere is $M_{\varphi}=P_{0, q} / g_{9}=1.03 \times 10^{6} \mathrm{~kg} / \mathrm{m}^{2}$. With a molecular mass of $m_{\varphi}=43.45 \mathrm{~g} / \mathrm{mol}$ that is $2.387 \times 10^{7} \mathrm{~mol} / \mathrm{m}^{2}$. The atmospheric composition is similar to that of Mars, with $96.5 \%$ molar fraction $\mathrm{CO}_{2}$. It has really astronomical amounts of $\mathrm{CO}_{2}$ (no pun intended). $96.5 \%$ of the mass is $2.3036 \times 10^{7} \mathrm{~mol} / \mathrm{m}^{2}$, about 5 orders of magnitude more than on Earth and 4 orders of magnitude more than on Mars. At such densities forbidden transitions in the spectrum, with small oscillator strengths, may start playing a role too. Non-linear effects, such as increased weight of the tails in the Lorentzian line shape (falling off quadratically with wavelength only) making the lines effectively broaden, may also become important.

When we look at reality, the greenhouse effect on Venus is enormous. Comparing the real temperature $T_{0, q}=737 \mathrm{~K}$ with the blackbody temperature based on the solar radiance and albedo, $\sigma T_{\mathrm{bb}, q}^{4}=\left(1-a_{\varphi}\right) W_{\varphi} / 4=0.23 \times\left(2601.3 \mathrm{~W} / \mathrm{m}^{2}\right) / 4$, of only $226.6 \mathrm{~K}$, we determine that the emissivity of Venus is very small: $\epsilon=[(226.6 \mathrm{~K}) /(737 \mathrm{~K})]^{4}=0.00894$. For these values we see that the radiation no longer comes from the surface at all, but from the atmosphere, instead, that is very opaque with an optical depth $\tau$ of about 81 (see Figure 12). Venus connects to the universe at a high altitude in the atmosphere. The heat finds it way to the surface by thermodynamic means, resulting in a high surface temperature.

\subsection{Geological Time Scales}

Another experiment nature throws at us is the geological time scale, from times long before humans appeared on this planet. Figure 15 shows these data and it is 


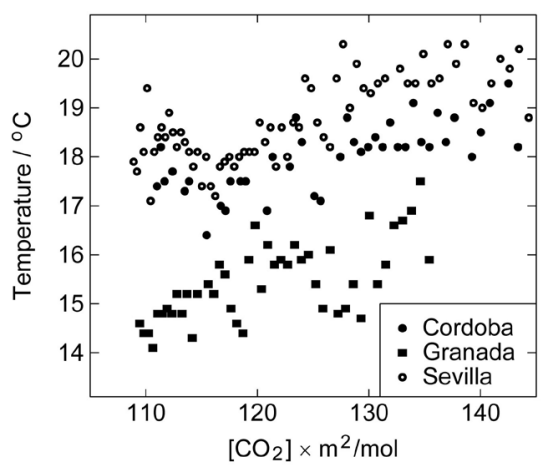

Figure 14. Relation between temperature and carbon content above the nearby cities of Sevilla, Córdoba and Granada in the south of Spain. $\mathrm{R}^{2}=0.21$.

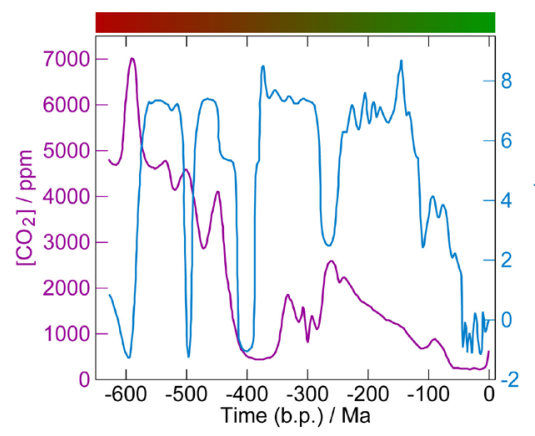

(a)

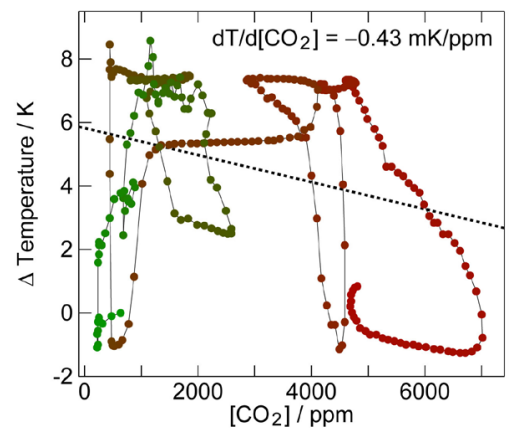

(b)

Figure 15. (a) Geological time scale data of temperature (blue curve) and $\left[\mathrm{CO}_{2}\right]$ (purple curve); (b) A correlation curve shows an absence of correlation between the two on this time scale. Plots made with the help of WebPlotDigitizer 4.2 [29] from a plot of Nasif Nahle [30].

obvious that in this time scale there is no correlation between carbon-dioxide concentrations in the air and surface temperatures. In fact, a fitting to the data results in a correlation of $\mathrm{d} T / \mathrm{d}\left[\mathrm{CO}_{2}\right]=-0.43 \mathrm{mK} / \mathrm{ppm}$, which is probably accidental, because we do not know any theory that might explain an inverse correlation between the two quantities.

A similar non-correlation we see in the Holocene data of Greenland, see Figure 16. Here a (pseudo)correlation of $\mathrm{d} T / \mathrm{d}\left[\mathrm{CO}_{2}\right]=-43 \mathrm{mK} / \mathrm{ppm}$ is found, two orders-of-magnitude more than in the paleontological data of Figure 15.

These results undermine the hypothesis that only $\mathrm{CO}_{2}$ is climate forcing, something that some climatologists claim.

\section{Other Effects: Feedback, Delay, Water}

Let us now go back to the figure of contemporary data of temperature anomaly vs. $\left[\mathrm{CO}_{2}\right]$ (Figure $2(\mathrm{~b})$ ). It was shown there that at $320 \mathrm{ppm}$ the anomaly was $-0.18 \mathrm{~K}$ and at $400 \mathrm{ppm}$ about $0.7 \mathrm{~K}$. We found an empirical relation equal to $\mathrm{d} T / \mathrm{d}\left[\mathrm{CO}_{2}\right]=10.2 \mathrm{mK} / \mathrm{ppm}$. Comparing it to the theoretical value of 3.3 $\mathrm{mK} / \mathrm{ppm}$ given before (Equation (60)), if we want to attribute the observed correlation to $\mathrm{CO}_{2}$, we have to include some kind of feedback (the increased 


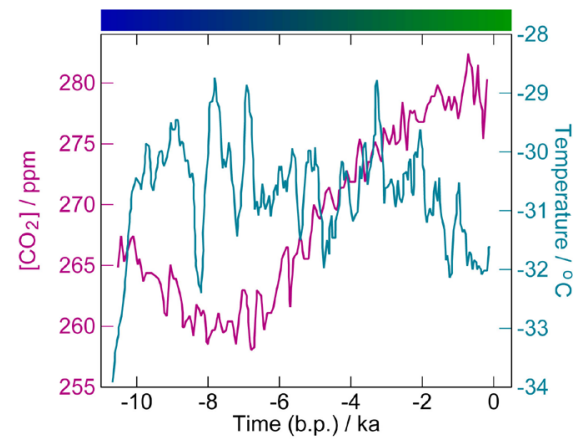

(a)

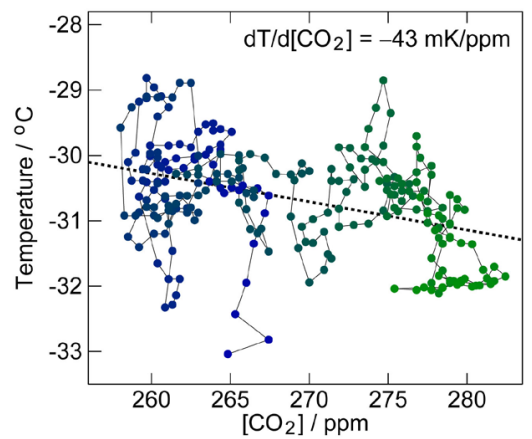

(b)

Figure 16. (a) (Holocene) ten-thousand-year time scale data of temperature (blue curve) and $\left[\mathrm{CO}_{2}\right]$ (purple curve) of Greenland; (b) A correlation curve shows an absence of correlation between the two on this time scale. Plots made with the help of WebPlotDigitizer 4.2 [29] from a plot of based on data of GISP 2 (temperature) and EPICA C $\left(\mathrm{CO}_{2}\right)$, found at Climate4You [31].

temperature again increases $\left[\mathrm{CO}_{2}\right]$, etc), or any other effect, for instance that the $\mathrm{CO}_{2}$ not only increases the absorption by that molecule, but also increases the amount of other substances in the air, thus increasing the effective absorption cross-section even more. If we call the climate sensitivity above in Equation (60) the open-loop response, $s$, the feedback $\beta$ (multiply output $T$ by $\beta$ and add it to the $\left[\mathrm{CO}_{2}\right]$ input; $\left.T \rightarrow\left[\mathrm{CO}_{2}\right]\right)$, we get an overall response function of

$$
\frac{\mathrm{d} T}{\mathrm{~d}\left[\mathrm{CO}_{2}\right]}=\frac{s}{1-s \beta} \text {. }
$$

The observed value in contemporary data shown in Figure 2(b), $10.2 \mathrm{mK} / \mathrm{ppm}$, thus yields a value of $\beta=0.205 \mathrm{ppm} / \mathrm{mK}$. The ice core data, on the other hand, show $95 \mathrm{mK} / \mathrm{ppm}$ correlation (Table 2). And this value yields a $\beta=0.293 \mathrm{ppm} / \mathrm{mK}$. Both allegedly being governed by the same process (greenhouse effect), it is inexplicable that the correlation factors are different. (Time delays cannot explain it, as will be shown later). These responses and feedback factors are inexplicably different; while the physics is allegedly the same. Both $\beta$ s are also incommensurate with the observed real responses $T \rightarrow\left[\mathrm{CO}_{2}\right]$ of the atmosphere in El Niño and La Niña years, which are of the order of $0.001 \mathrm{ppm} / \mathrm{mK}$ [6], orders of magnitude below the theoretical $\beta$ s needed to explain things, although delay can play a crucial factor here. Moreover, the $\beta$ s above are close to the critical Barkhausen value of infinite response, which occurs when $s \beta=1$, at $\beta=0.303 \mathrm{ppm} / \mathrm{mK}$, or any value beyond it. 0.293 is too close to 0.303 for comfort. We thus exclude feedback, $\left[\mathrm{CO}_{2}\right] \leftrightarrow T$, as a phenomenon to explain the observations. And we thus reject on basis of empirical data the hypothesis of the greenhouse effect as an explanation for the observed correlations between $T$ and $\left[\mathrm{CO}_{2}\right]$ [6]. We cannot determine empirically the magnitude of the greenhouse effect, only that it is too small to explain the observations.

The alternative explanation for the correlations is called Henry's Law mentioned before $\left(T \rightarrow\left[\mathrm{CO}_{2}\right]\right)$, and this fully explains all data [1], we can thus not 
call it a feedback effect, when the feed-forward effect (greenhouse effect) is negligible compared to the feed-back effect (Henry's Law); the correlation-on all time scales-can easily be explained by only the latter (HL), without the need of feedback (GHE, at any magnitude), and cannot be explained by the former (GHE), not even with HL-feedback or any other reasonable feedback mechanism. Henry's Law was estimated to be $0.010 \mathrm{ppm} / \mathrm{mK}$ (Table 2). We see that if we want to use Henry's Law as feedback mechanism in the greenhouse-effect model, a factor $24-34$ is missing. We do not know of other reasonable feedback mechanisms.

We may also think of secondary effects, such as methane-outgassing of melting permafrost regions or increased amounts of water in the air, $\left[\mathrm{CO}_{2}\right] \rightarrow T \rightarrow$ $\left[\mathrm{H}_{2} \mathrm{O}\right]$, to be discussed in a section below. This kind of effects is also often mentioned as "feedback" in literature, though technically speaking they are not, because they do not result in increments in the original signal, $\left[\mathrm{CO}_{2}\right]$, but only have secondary effects other than the primary one of increasing directly the temperature. As in $\left[\mathrm{CO}_{2}\right] \rightarrow T \rightarrow\left[\mathrm{H}_{2} \mathrm{O}\right] \rightarrow T$.

\subsection{Delay}

We might think that the atmosphere did not have time yet to reach the new equilibrium. The observed effects are then always less than the one calculated. For the greenhouse effect, however, we need to explain that the signal is larger than theory predicts. Considering the fact that our calculations may be wrong, we can, still, make an estimation about how long it takes to reach the equilibrium, and turn the observed short-term contemporary $10.2 \mathrm{mK} / \mathrm{ppm}$ into the observed long-term $95 \mathrm{mK} / \mathrm{ppm}$.

The specific heat capacity of air is $c_{\mathrm{p}}=1.51 \mathrm{~kJ} / \mathrm{K} \cdot \mathrm{kg}$. The pressure is 1013.2 $\mathrm{Pa}$, so the mass density is $M=P_{0} / g=10.3 \times 10^{3} \mathrm{~kg} / \mathrm{m}^{2}$. We found a radiative forcing of $w=8.6 \mathrm{~mW} / \mathrm{m}^{2}$ per ppm (Equation (61)), and a temperature effect of $\Delta T / \Delta\left[\mathrm{CO}_{2}\right]=3.3 \mathrm{mK} / \mathrm{ppm}$ (Equation (60)). The characteristic temperature adjustment time of just the atmosphere alone is then

$$
\tau_{\mathrm{GHE}}=\frac{\Delta T / \Delta\left[\mathrm{CO}_{2}\right] \times M \times c_{\mathrm{p}}}{w}=46 \mathrm{~d} .
$$

That is about a month and a half, a value very similar to the one we found empirically in the phase shift of yearly-periodic solar radiation and temperature data, namely about 1.2 months [32] and a simple relaxation analysis of daily temperature variations which gives 23 days [1]. We can thus exclude any substantial delay effects in the greenhouse effect $\left[\mathrm{CO}_{2}\right] \rightarrow T$ on the time scales of the contemporary and ice-core-drilling datasets, 60 a and $600 \mathrm{ka}$, respectively. On the other hand, we can expect long delays between $T$ and $\left[\mathrm{CO}_{2}\right]$ in the framework of Henry's Law. Imagine the atmosphere warms up for some reason (maybe solar activity). This warmed up air must then heat up the relevant layer of the ocean and expose this layer to the surface where the surplus $\mathrm{CO}_{2}$ can outgas. This surface mixing layer is about $300 \mathrm{~m}$ thick, it has thus a mass density of 
$M_{\text {ocean }}=3 \times 10^{5} \mathrm{~kg} / \mathrm{m}^{2}$, which has a specific heat of $c_{\text {water }}=4.12 \mathrm{~kJ} / \mathrm{kg} \cdot \mathrm{K}$. We can assume the bottleneck is the heating up itself and diffusion and rotation of the water are rather fast. Estimating the maximum extra heat the atmosphere can exchange with the water to be governed by Stefan-Boltzmann (Equation (9), $\Delta T=1 \mathrm{~K}$ will give $w=5.4 \mathrm{~W} / \mathrm{m}^{2}$ extra radiation), we find an adjustment time of

$$
\tau_{\mathrm{HL}}=\frac{\Delta T \times c_{\text {water }} \times M_{\text {ocean }}}{w}=7.2 \mathrm{a},
$$

which is commensurate the data observed in ice-core drillings. If the bottleneck is another process, like the the thermohaline circulation, the characteristic time may even be much longer, in the order of thousands of years.

\subsection{Water/Albedo}

The secondary effect of, for instance, water evaporation is very difficult to determine, but we can make an estimate that the temperature causes an increase of amount of water proportional to the saturation levels, and no extra clouds are formed that might block out incoming solar radiation (albedo effect). The Engineering Toolbox, once again, supplies the data for this calculation [33], see Figure 17: $1.0 \mathrm{~K}$ temperature rise might result in $6 \%$ extra water in the air. Since $95 \%$ of the greenhouse effect is caused by water, we might expect an increase of the absorption coefficient by $5.7 \%$, and if $3.62 \%$ resulted in $1.0 \mathrm{~K}$ (of total $T_{0}-T_{\mathrm{bb}}=288 \mathrm{~K}-245 \mathrm{~K}$ ), $5.7 \%$ will result in something like $1.57 \mathrm{~K}$, which will result in even more water, etc. A positive-feedback loop of $1.57 \mathrm{~K} / \mathrm{K}$ obviously will spin out of control. The $95 \%$ effect of water makes sense, also in the thermodynamic-radiative theory, since water is still active in the part of the emission spectrum that is still open.

It is clear that this analysis is incorrect, since it is a run-away series. Any increase in temperature $\delta T$ or atmospheric water content $\delta\left[\mathrm{H}_{2} \mathrm{O}\right]$, however small, and whatever the cause is (even if not $\mathrm{CO}_{2}$ ), will result in a run-away scenario: $\delta T$ becomes $1.57 \delta T$ becomes $1.57^{2} \delta T$, etc. The fact that this
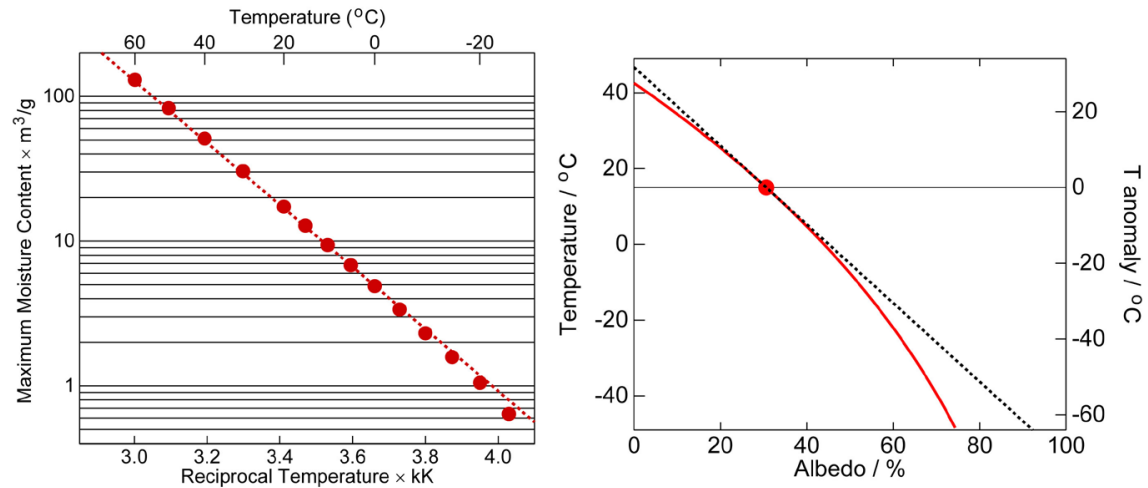

Figure 17. Left: Saturation water content in atmosphere as a function of temperature $(1000 / T)$. Source: Ref. [33]. Right: Effect of albedo on temperature, the sensitivity shown by the dashed curve is $\mathrm{d} T / \mathrm{d} a=-103.8 \mathrm{~K}$. 
runaway scenario is never observed proves the thesis that the feedback between temperature and water is negative instead. We can guess at what the process of negative feedback is, but we suspect increased cloud cover: reflection of incoming solar radiation by the clouds increases the albedo. Increased water content decreases the temperature and removes water from the air.

Whatever the phenomenon responsible for the warming of the planet, the final temperature is in any case proportional to the fourth power of incoming solar radiation: $T_{0} \propto \sqrt[4]{(1-a) S / \sigma}$, we thus easily find an albedo sensitivity of

$$
\frac{\mathrm{d} T_{0}}{\mathrm{~d} a}=-\frac{T_{0}}{4(1-a)}=-103.8 \mathrm{~K} .
$$

The climate is highly sensitive to albedo changes: A little more than a degree for every percent of change of $a$. The albedo dependence and this climate sensitivity are shown in Figure 17. Water surfaces have a visible albedo of 0.06. Land (from wet sand, to dry soil, to desert) has an albedo in the order of 0.25 . Seas cover $71 \%$ of the planet, land the remaining $29 \%$. The average cloudless albedo would thus be ca. 0.115 . Clouds have an albedo of about 0.60 , and the coverage is thus about $41 \%$ to result in the Earth's albedo of 0.306 . From this follows a water sensitivity on albedo: If we increase water by $1 \%$ in the air, clouds will increase by $1 \%$ (in a linear analysis) and cover $0.41 \%$ more of the planet and cloudless area will be $0.41 \%$ less, increasing the albedo by $2.5 \times 10^{-3}$. That results in a temperature drop of $0.26 \mathrm{~K}$ :

$$
\left.\frac{\mathrm{d} T_{0}}{\mathrm{~d}\left[\mathrm{H}_{2} \mathrm{O}\right]}\right|_{\text {albedo }}=-260 \mathrm{mK} \text { per } \%
$$

The observed cloud cover variations are in the order of three percent [34], with less coverage in the recent years. Also, reconstructed albedo varied by a couple of percent [35] with a radiative forcing variation of about $5 \mathrm{~W} / \mathrm{m}^{2}$. This can thus explain the global temperature variations that are indeed of the order of half a degree to a degree. No variation of greenhouse effect is needed to explain the data, and Ockam's Razor would make us remove this phenomenon from the explanations of climate changes.

Water has still another effect, namely that of increasing the specific heat of air $\left(c_{\mathrm{p}}\right)$ and thus of the lapse rate $\left(\Gamma=-g / c_{\mathrm{p}}\right)$. Since the total amount of energy of the entire atmosphere is given by $c_{\mathrm{p}} M T_{0}$, the immediate effect of increasing $c_{\mathrm{p}}$ is decreasing the surface temperature. However, we need to do a full calculation to see what happens. Figure 9(b) shows a simulation of the Earth system, with $c_{\mathrm{p}}$ doubled. Maintaining the optical properties of the atmosphere (same optical depth, $\tau$ ), we can see that the emissivity of Earth increases, and that means that the surface is cooling down, according to Equation (58). We can also see that relatively more radiation comes from the atmosphere in this case, that must have heated up effectively.

In fact, upon analysis of these results we find that changing the specific heat $c_{\mathrm{p}}$ by $1 \%$ results in an increase of the planetary emissivity by $0.12 \%$, and thus a 
lowering of the temperature by $0.03 \%(86 \mathrm{mK})$. Or, in other words, a sensitivity of $\mathrm{d} \epsilon / \mathrm{d} c_{\mathrm{p}}=0.0481 \mathrm{~kg} \cdot \mathrm{K} / \mathrm{kJ}$. The climate sensitivity of planetary emissivity, from Equation (58), is $\mathrm{d} T_{0} / \mathrm{d} \epsilon=-T_{\mathrm{bb}} / 4 \epsilon \sqrt[4]{\epsilon}=-118.91 \mathrm{~K}$. Moreover, from Equation (34), we find that $c_{\mathrm{p}}$ depends on water content in the atmosphere equal to $\mathrm{d} c_{\mathrm{p}} /(\mathrm{d} x / x)=5.10 \times 10^{-3} \mathrm{~kJ} / \mathrm{kg} \cdot \mathrm{K}$ per $\%$ increase of $\left[\mathrm{H}_{2} \mathrm{O}\right]$ in the atmosphere. Therefore, the sensitivity of climate to water, through the lapse rate mechanism, is

$$
\left.\frac{\mathrm{d} T_{0}}{\mathrm{~d}\left[\mathrm{H}_{2} \mathrm{O}\right]}\right|_{\text {lapserate }}=\frac{\mathrm{d} T_{0}}{\mathrm{~d} \epsilon} \cdot \frac{\mathrm{d} \epsilon}{\mathrm{d} c_{\mathrm{p}}} \cdot \frac{\mathrm{d} c_{\mathrm{p}}}{\mathrm{d}\left[\mathrm{H}_{2} \mathrm{O}\right]}=-29 \mathrm{mK} \text { per } \% .
$$

This is an order of magnitude less than the climate sensitivity on water through the albedo effect.

We also found an open-loop temperature water sensitivity $d\left[\mathrm{H}_{2} \mathrm{O}\right] / \mathrm{d} T$ of $6 \% / \mathrm{K}$ assuming capture cross sections change temperature. This effect of rising temperature $1 \mathrm{~K}$ would thus be compensated by a feedback of lowering the temperature by $6 \% \times(0.29 \mathrm{~K} / \%)=1.74 \mathrm{~K}$. Added to this the positive feedback loop greenhouse effect of $1.57 \mathrm{~K} / \mathrm{K}$ found above, the total feedback would be slightly negative. Adding water to the air then lowers the temperature.

Summarizing this part: Water has several effects. First it will change the specific heat of the air $\left(c_{\mathrm{p}}\right)$ and thus the lapse rate of the atmosphere, Equation (36). This will slightly lower the temperature at the surface. As a secondary effect, it will increase dramatically the absorption cross section for infrared, and this will change the ground temperature $T_{0}$. The feedback effect of water on outgoing radiation is beyond the Barkhausen criterion and thus, any addition of water to the atmosphere, however tiny, would result in a run-away scenario. However, this effect is fully canceled by the albedo effect, resulting in a net negative effect of water on the temperature.

\section{Conclusions}

We have analyzed here the greenhouse effect, using fully analytical techniques, without reverting anywhere to finite-elements calculations. This gave important insight into the phenomenon. An important conclusion is that the analysis in terms of radiation-balances-only cannot explain the situation in the atmosphere. In the extreme case, a differential equation of layers with absorption coefficients, etc., gave the same results as a much simpler 2-box mixed chamber model. However, the underlying assumptions in these calculations are not physical.

Therefore we set out to model the greenhouse effect $a b$ initio, and came up with the thermodynamic-radiation model. The atmosphere is close to thermodynamic equilibrium and based on that we can calculate where and how radiation is absorbed and emitted. This model can explain phenomenologically and analytically how big the effect of the atmosphere is, specifically Equations (56) and (58).

Continuing with the reasoning, we find that the alleged greenhouse effect cannot explain the empirical data-orders of magnitude are missing. There where 
Henry's Law-outgassing of oceans-easily can explain all observed phenomena.

Moreover, the greenhouse hypothesis-as presented here-cannot explain the atmosphere on Mars, nor can it explain the geological data, where no correlation between $\left[\mathrm{CO}_{2}\right]$ and temperature is observed. Nor can it explain why a different correlation is observed in contemporary data of the last 60 years compared to historical data (600 thousand years). We thus reject the anthropogenic global warming (AGW) hypothesis, both on basis of empirical grounds as well as a theoretical analysis.

\section{Conflicts of Interest}

The author declares no conflicts of interest regarding the publication of this paper. This research was not funded.

\section{References}

[1] Stallinga, P. (2018) Signal Analysis of the Climate: Correlation, Delay and Feedback. Journal of Data Analysis and Information Processing, 6, 30-45. https://doi.org/10.4236/jdaip.2018.62003

[2] Stallinga, P. and Khmelinskii, I. (2017) Perils and Pitfalls of Empirical Forecasting. European Scientific Journal, 13, 18-46. https://doi.org/10.19044/esj.2017.v13n18p18

[3] Stallinga, P. and Khmelinskii, I. (2017) Negative Feedback in the Polar Ice System. Atmospheric and Climate Sciences, 7, 76. https://doi.org/10.4236/acs.2017.71007

[4] Chen, C., et al. (2019) China and India Lead in Greening of the World through Land-Use Management. Nature Sustainability, 2, 122-129. https://doi.org/10.1038/s41893-019-0220-7

[5] Ångström, K. (1900) Ueber die Bedeutung des Wasserdampfes und der Kohlensäure bei der Absorption der Erdatmosphäre. Annalen der Physik, 308, 720-732. https://doi.org/10.1002/andp.19003081208

[6] Stallinga, P. and Khmelinskii, I. (2018) Analysis of Temporal Signals of Climate. Natural Science, 10, 393-403. https://doi.org/10.4236/ns.2018.1010037

[7] Soon, W., Connolly, R. and Connolly, M. (2015) Re-Evaluating the Role of Solar Variability on Northern Hemisphere Temperature Trends since the 19th Century. Earth Science Reviews, 450, 409-452. https://doi.org/10.1016/j.earscirev.2015.08.010

[8] Integrated Publishing. U.S. Standard Atmosphere Heights and Temperatures. http://meteorologytraining.tpub.com/14269/css/14269_75.htm

[9] Myhre, G., et al. (1998) New Estimates of Radiative Forcing Due to Well Mixed Greenhouse Gases. Geophysical Research Letters, 25, 2715-2718. https://doi.org/10.1029/98GL01908

[10] Volokin, D. and ReLlez, L. (2014) On the Average Temperature of Airless Spherical Bodies and the Magnitude of Earth's Atmospheric Thermal Effect. SpringerPlus, 3, 723. https://doi.org/10.1186/2193-1801-3-723

[11] The Engineering Toolbox. Air-Specific Heat at Constant Pressure and Varying Temperature. https://www.engineeringtoolbox.com/air-specific-heat-capacity-d_705.html

[12] The Engineering Toolbox. Heating Humid Air. https://www.engineeringtoolbox.com/heatinghumid-air-d_693.html

[13] The Engineering Toolbox. Specific Heat and Individual Gas Constant of Gases. 
http://www.engineeringtoolbox.com/specific-heat-capacity-gases-d_159.html

[14] The Engineering Toolbox. U.S. Standard Atmosphere. https://www.engineeringtoolbox.com/standard-atmosphere-d_604.html

[15] Cotton, D. (2013) Planetary Core and Surface Temperatures. https://doi.org/10.2139/ssrn.2876905

[16] Connolly, M. and Connolly, R. (2014) The Physics of the Earth's Atmosphere III. Pervective Power. Open Peer Review Journal, 25, ver. 0.1. http://oprj.net/articles/atmosphericscience/25

[17] Holzner, S. (2005) Physics for Dummies. For Dummies.

[18] Hieb, M. (2003) Global Warming: A Closer Look at the Numbers. https://www.geocraft.com/WVFossils/greenhouse_data.html

[19] Fleming, R.J. (2018) An Updated Review about Carbon Dioxide and Climate Change. Environmental Earth Sciences, 77, 262. https://doi.org/10.1007/s12665-018-7438-y

[20] Gradsthteyn, I.S. and Ryzhik, I.M. (2000) Table of Integrals, Series and Products. 6th Edition, Academic Press, Cambridge, 3.382.

[21] Abramowitz, M. and Stegun, I.A. (1972) Handbook of Mathematical Functions. National Bureau of Standards, 6.5.2.

[22] NASA. Mars Fact Sheet. https://nssdc.gsfc.nasa.gov/planetary/factsheet/marsfact.html

[23] The Engineering Toolbox. Molecular Weight-Common Substances. https://www.engineeringtoolbox.com/molecular-weight-gas-vapor-d_1156.html

[24] NASA. Venus Fact Sheet. https://nssdc.gsfc.nasa.gov/planetary/factsheet/venusfact.html

[25] University of Oxford. Department of Physics. Atmospheric Infrared Spectrum Atlas. http://eodg.atm.ox.ac.uk/ATLAS

[26] Clive Best. Doubling $\mathrm{CO}_{2}$ and Basic Physics. http://clivebest.com/blog/?p=1169

[27] Jacob, D.J. (1999) Introduction to Atmospheric Chemistry. Princeton University Press, Princeton.

[28] NOAA. National Centers for Environmental Information. Climate Data Online: Dataset Discovery. https://www.ncdc.noaa.gov/cdo-web/datasets

[29] Ankit Rohatgi. WebPlotDigitizer. https://automeris.io/WebPlotDigitizer

[30] Nasif Nahle. Geologic Global Climate Changes. http://www.biocab.org/carbon_dioxide_geological_timescale.html

[31] Climate4You. Temperature since 10700 0BP with $\mathrm{CO}_{2}$ from EPICA DomeC. http://www.climate4you.com/images/GISP2\%20TemperatureSince10700\%20BP\%20 with\%20CO2\%20from\%20EPICA\%20DomeC.gif

[32] Khmelinskii, I. and Stallinga, P. (2014) Application of Signal Analysis to the Climate. International Scholarly Research Notices, 2014, Article ID: 161530. https://doi.org/10.1155/2014/161530

[33] The Engineering Toolbox. U.S. Standard Atmosphere. https://www.engineeringtoolbox.com/maximum-moisture-content-air-d_1403.html

[34] International Satellite Cloud Climatology Project. Cloud Analysis-Part 1: Climatology of Global Cloud and Surface Properties: Cloud Amount. https://isccp.giss.nasa.gov/analysis/climanall.html

[35] Pallé, E., et al. (2004) Changes in Earth's Reflectance over the Past Two Decades. Science, 304, 1299-1301. https://doi.org/10.1126/science.1094070 